\title{
Analysis of 2-dimensional transient problems for linear elastic and piezoelectric structures using the consecutive-interpolation quadrilateral element (CQ4)
}

\author{
Tinh Quoc Bui ${ }^{\text {a, }}{ }^{\text {, }}$, Du Dinh Nguyen ${ }^{\mathrm{b}}$, Xiaodong Zhang ${ }^{\mathrm{c}}$, Sohichi Hirose ${ }^{\mathrm{a}}$, \\ Romesh C. Batra ${ }^{\mathrm{d}}$ \\ a Department of Mechanical and Environmental Informatics, Tokyo Institute of Technology, 2-12-1-W8-22, Ookayama, Meguro-ku, Tokyo, 152-8552, Japan \\ ${ }^{\mathrm{b}}$ Department of Civil Engineering, Lac-Hong University, Dong Nai Province, Viet Nam \\ c State Key Laboratory of Bridge Engineering Structural Dynamics, China Merchants Chongquig Communications Research \& Design Institute Co. Ltd., Chong \\ Qing, PR China \\ ${ }^{\mathrm{d}}$ Department of Biomedical Engineering and Mechanics, M/C 0219, Virginia Polytechnic Institute and State University, Blacksburg, VA, 24061, USA
}

\section{A R T I C L E I N F O}

Article history:

Received 16 July 2015

Accepted 11 January 2016

Available online 28 January 2016

\section{Keywords:}

Elastodynamics

Quadrilateral element

Vibration

\begin{abstract}
A B S T R A C T
The recently developed consecutive-interpolation procedure (CIP) based 4-node quadrilateral element (CQ4) is used to study free and forced 2-dimensional vibrations of elastic and piezoelectric structures of complex geometries. For some of these problems results have also been computed with the standard quadrilateral element under the constraint that the two approaches have the same number of degrees of freedom. In each case it is found that the numerical solution using the CQ4 element has better accuracy than that found with the standard quadrilateral element, and these solutions agree well with results available in the literature. The main difference between the CIP basis functions and those for the traditional 4-node quadrilateral element is that the former incorporate both nodal values and averaged gradients of the trial solution at the nodes and the latter only the nodal values. However, for both sets of basis functions only nodal values appear as unknowns in the matrix formulation of the problem.
\end{abstract}

๑ 2016 Elsevier Masson SAS. All rights reserved.

\section{Introduction}

The development of a consecutive-interpolation 4-node quadrilateral element (CQ4) for stress analysis of 2-dimensional structures based on the consecutive-interpolation procedure (CIP) of Zheng et al. (2010) was recently presented in Bui et al. (2014). The basis functions for the CQ4 element are constructed in two stages. First the same interpolation functions as in the conventional finite element method (FEM) for the 4-node quadrilateral element are constructed. Subsequently, they are extended to include both the nodal values and the averaged values of gradients of the unknown function at the nodes. The main motivation of the CIP is to make the trial solution and its derivatives continuous across interelement boundaries. This should improve the accuracy of the computed gradients of the trial solution and avoid using smoothing techniques generally employed during the post-processing process.

\footnotetext{
* Corresponding author. Tel.: +81 357343587.

E-mail address: tinh.buiquoc@gmail.com (T.Q. Bui).
}

Numerical solution of several elastostatic problems presented in Zheng et al. (2010), Bui et al. (2014) and Kang et al. (2015) have demonstrated that these basis functions for the CQ4 element yield, for the same number of degrees of freedom (DoFs) as for the traditional Q4 element, more accurate values of the trial solution that have better convergence rates than those for the standard Q4 element. Furthermore, good values of stresses at nodes can be found without using smoothing operations. The shape functions for the CQ4 element are linearly independent, satisfy the partition of unity, possess Kronecker-delta function property, and alleviate the volumetric locking issue for incompressible materials. The CQ4 element can be easily implemented in existing software since the numerical solution procedure for the two elements is essentially the same.

Here we extend the applicability of the CQ4 element basis functions to the analysis of 2-dimensional (either plane stress or axisymmetric) free and forced vibration problems, and show that indeed these basis functions give improved values of natural frequencies and mode shapes for structures of complex geometry. For forced vibration problems they provide accurate values of nodal 
displacements and their transient responses. When solving these problems, the coupled ordinary differential equations obtained by the FEM are numerically integrated by using both implicit unconditionally stable and explicit conditionally stable methods. We have also extended their applicability to study free vibrations of piezoelectric structures for which the electric and the mechanical fields are coupled. It is shown that the numerical results obtained with the CQ4 element agree well with those reported in the literature and obtained by such methods as the boundary element method (Manolis and Beskos, 1988), the FEM (Petyt, 2010; Miranda et al., 2008; Dai and Liu, 2007), mesh-free methods (Bui et al., 2011a,b; 2013; Bui and Nguyen, 2011; Kosta and Tsukanov, 2014; Sadeghirad et al., 2009; Cui et al., 2010; Gu and Liu, 2005), and the isogeometric analysis (Valizadeh et al., 2013; Cottrell et al., 2006).

The rest of the paper is organized as follows. The formulation of a linear elastodynamic problem is given in Section 2 wherein we also briefly review the derivation of the discrete equations and the basis functions for the CQ4 element. Frequencies and the associated mode shapes for free vibrations of five structures are presented in Section 3. Forced vibrations of a cantilever beam with timedependent tangential tractions applied on its unclamped edge, and those of an L-shaped bracket with a uniformly distributed time-dependent pressure applied on the top surface are studied in Section 4. In Section 5, we provide results of free vibration of a piezoelectric disc and a transducer. Conclusions of this work are summarized in Section 6.

\section{Formulation of the problem}

\subsection{Problem statement}

We analyze free and forced vibrations of a homogeneous, isotropic and linear elastic body occupying a planar domain $\Omega$ with boundary $\Gamma$. In a rectangular Cartesian coordinate system 2dimensional deformations of the body are governed by

$\mathbf{L}^{\mathrm{T}} \boldsymbol{\sigma}=\mathbf{b}-\rho \ddot{\mathbf{u}}-\eta \dot{\mathbf{u}}$ in $\Omega$

where the partial differential operator matrix $\mathbf{L}$ is defined by

$\mathbf{L}^{\mathrm{T}}=\left[\begin{array}{ccc}\frac{\partial}{\partial x} & 0 & \frac{\partial}{\partial y} \\ 0 & \frac{\partial}{\partial y} & \frac{\partial}{\partial x}\end{array}\right]$

Furthermore, $\mathbf{u}, \dot{\mathbf{u}}$ and $\ddot{\mathbf{u}}$ are, respectively, the displacement, the velocity and the acceleration of a material particle, $\rho$ the mass density, $\eta$ the damping coefficient, $\sigma^{T}=\left\{\sigma_{x x}, \sigma_{y y}, \sigma_{x y}\right\}$ the stress tensor, and $\mathbf{b}$ the body force field. Eqs. (1) and (2) are supplemented by the following essential and natural boundary conditions given by Eqs. (3a) and (3b), respectively.

$\mathbf{u}=\overline{\mathbf{u}}$ on $\Gamma_{u}$

$\mathbf{t}=\mathbf{n}^{\mathrm{T}} \boldsymbol{\sigma}=\overline{\mathbf{t}}$ on $\Gamma_{t}$

Here $\overline{\mathbf{u}}$ is the prescribed displacement at points on the boundary $\Gamma_{u}$, $\overline{\mathbf{t}}$ the surface traction prescribed on $\Gamma_{t}$,

$\mathbf{n}=\left[\begin{array}{cc}n_{x} & 0 \\ 0 & n_{y} \\ n_{y} & n_{x}\end{array}\right]$ where $n_{x}$ and $n_{y}$ are components of the unit outward normal $\mathbf{n}$ to the boundary, and $\Gamma=\Gamma_{u} \cup \Gamma_{t}$. The pertinent initial conditions are

$\mathbf{u}\left(\mathbf{x}, t_{0}\right)=\mathbf{u}_{0}(\mathbf{x}), \mathbf{x} \in \Omega$

$\dot{\mathbf{u}}\left(\mathbf{x}, t_{0}\right)=\mathbf{v}_{0}(\mathbf{x}), \mathbf{x} \in \Omega$

where $\mathbf{u}_{0}$ and $\mathbf{v}_{0}$ are displacements and velocities at the initial time $t_{0}$, respectively.

\subsection{Weak-form and discrete equations}

We use the principle of virtual work to derive coupled ordinary differential equations (ODEs) governing transient deformations of the body. Denoting by $\delta \mathbf{u}$ and $\delta \boldsymbol{\varepsilon}$, respectively, the virtual displacement and the virtual strain fields in the body, the principle of virtual work requires that

$\int_{\Omega} \delta \boldsymbol{\varepsilon}^{T} \boldsymbol{\sigma} d \Omega-\int_{\Omega} \delta \mathbf{u}^{T}[\mathbf{b}-\rho \ddot{\mathbf{u}}-\eta \dot{\mathbf{u}}] d \Omega-\int_{\Gamma_{t}} \delta \mathbf{u}^{T} \overline{\mathbf{t}} d \Gamma=0$

for every time $t$. We note that $\delta \mathbf{u}$ vanishes on $\Gamma_{u \text {. }}$

The domain $\Omega$ is discretized into a FE mesh composed of 4-node quadrilateral elements. However, basis functions used herein are different from those in the traditional FEM. The virtual displacements, $\delta \mathbf{d}$, of nodes that lie on the boundary $\Gamma_{u}$ vanish. The virtual displacements and the corresponding virtual strains in an element are related to the virtual nodal displacement, $\delta \mathbf{d}$, as follows:

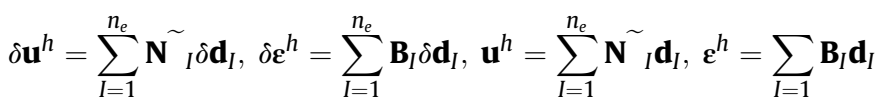

where $n_{e}$ is the number of nodes on an element, $\mathbf{d}_{I}=\left[u_{I}, v_{I}\right]^{\mathrm{T}}$ the nodal displacement vector, $\tilde{\mathbf{N}}_{I}=\left[\begin{array}{cc}\tilde{N}_{I} & 0 \\ 0 & \tilde{N}_{I}\end{array}\right]$ the CQ4 shape function matrix defined in the next subsection,

$\mathbf{B}_{I}=\mathbf{L} \tilde{\mathbf{N}}_{I}(\mathbf{x})=\left[\begin{array}{cc}\tilde{N}_{I, x} & 0 \\ 0 & \tilde{N}_{I, y} \\ \tilde{N}_{I, y} & \tilde{N}_{I, x}\end{array}\right]$

the displacement gradient matrix, and $\tilde{N}_{I, X}$ is the partial derivative of $\tilde{N}_{I}$ with respect to $x$. We note that the matrix $\mathbf{B}$ of derivatives of shape functions in Eq. (8) differs from that of the standard FEM because of the difference in the support domains of the shape function of a node. The support domain for the CQ4 element is, in general, larger than that for the conventional Q4 element. For a typical element, the explicit expression for the matrix $\mathbf{B}$ is

$\mathbf{B}_{e}=\left[\begin{array}{cccccccccccc}\frac{\partial \tilde{N}_{1}}{\partial x} & \frac{\partial \tilde{N}_{2}}{\partial x} & \cdots & \frac{\partial \tilde{N}_{\xi}}{\partial x} & \cdots & \frac{\partial \tilde{N}_{n_{s}}}{\partial x} & 0 & 0 & \cdots & 0 & \cdots & 0 \\ 0 & 0 & \cdots & 0 & \cdots & 0 & \frac{\partial \tilde{N}_{1}}{\partial y} & \frac{\partial \tilde{N}_{2}}{\partial y} & \cdots & \frac{\partial \tilde{N}_{\xi}}{\partial y} & \cdots & \frac{\partial \tilde{N}_{n_{s}}}{\partial y} \\ \frac{\partial \tilde{N}_{1}}{\partial y} & \frac{\partial \tilde{N}_{2}}{\partial y} & \cdots & \frac{\partial \tilde{N}_{\xi}}{\partial y} & \cdots & \frac{\partial \tilde{N}_{n_{s}}}{\partial y} & \frac{\partial \tilde{N}_{1}}{\partial x} & \frac{\partial \tilde{N}_{2}}{\partial x} & \cdots & \frac{\partial \tilde{N}_{\xi}}{\partial x} & \cdots & \frac{\partial \tilde{N}_{n_{s}}}{\partial x}\end{array}\right]_{\left(3 \times 2 n_{s}\right)}$

with $1<\xi<n_{s}$ and $n_{s}>4$ Bui et al., (2014). 

law

The stresses are assumed to be related to the strains by Hooke's

$\boldsymbol{\sigma}=\mathbf{D} \varepsilon$

where for plane stress deformations

$\mathbf{D}=\frac{E}{1-\nu^{2}}\left[\begin{array}{ccc}1 & \nu & 0 \\ \nu & 1 & 0 \\ 0 & 0 & (1-\nu) / 2\end{array}\right]$

with $E$ being Young's modulus and $\nu$ Poisson's ratio for the material of the body. One can use the current formulation for studying plane strain problems by modifying the matrix D. However, here we study only plane stress problems.

Following the standard argument, the ODEs for nodal displacements can be written as

$\ddot{\mathbf{M d}}+\ddot{\mathbf{C d}}+\mathbf{K d}=\mathbf{f}$

where $\mathbf{M}, \mathbf{C}$, and $\mathbf{K}$, respectively, are the mass, the damping and the stiffness matrices, and $\mathbf{f}$ is the force vector. These matrices are given by

$\mathbf{M}_{I J}=\sum_{e} \int_{\Omega_{e}} \tilde{\mathbf{N}}_{I}^{T} \boldsymbol{\rho} \tilde{\mathbf{N}}_{J} d \Omega$

$\mathbf{C}_{I J}=\sum_{e} \int_{\Omega_{e}} \tilde{\mathbf{N}}_{I}^{T} \boldsymbol{\eta} \tilde{\mathbf{N}}_{J} d \Omega$

$\mathbf{K}_{I J}=\sum_{e} \int_{\Omega_{e}} \mathbf{B}_{I}^{T} \mathbf{D} \mathbf{B}_{J} d \Omega$

$\mathbf{f}_{I}=\sum_{e} \int_{\Omega_{e}} \widetilde{\mathbf{N}}_{I}^{T} \mathbf{b}_{I} d \Omega+\sum_{e} \int_{I_{t} \cap \Omega_{e}} \widetilde{\mathbf{N}}_{I}^{T} \overline{\mathbf{t}}_{I} d \Gamma$

\subsection{The CQ4 shape functions and their properties}

The formulation of the CQ4 element and its basis functions was presented in detail by Bui et al. (2014). For the sake of completeness, we briefly describe here the CQ4 element. Assume that $\mathbf{x}=(x, y)$ is a point in the quadrilateral element with nodes $i, j, k, m$ as illustrated in Fig. 1. We denote by $S_{i}, S_{j}, S_{k}$ and $S_{m}$ elements that share nodes $i, j, k$ and $m$, respectively. In CQ4 element, the supporting nodes for the point $\mathbf{x}$ include all nodes of elements $S_{i}, S_{j}, S_{k}$ and $S_{m}$. Thus the support domain for point $\mathbf{x}$ is much larger than that in the standard FEM, and the trial solution at point $\mathbf{x}$ is assumed to be given by

$u^{h}(\mathbf{x})=\sum_{l=1}^{n_{s}} \tilde{N}_{l}(\mathbf{x}) d_{l}=\tilde{\mathbf{N}}(\mathbf{x}) \mathbf{d}$

where the consecutive-interpolation shape functions are given by
Here $d_{l}$ denotes the nodal displacement vector, $N_{l}^{[i]}$ is the vector of shape functions at node $i$, and $n_{s}$ is the total number of supporting nodes for the point $\mathbf{x}$. The average value of the derivative of the shape functions at node $i$ is written as follows:

$\begin{aligned} \bar{N}_{l, x}^{[i]} & =\sum_{e \in S_{i}}\left(w_{e} N_{l, x}^{[i][e]}\right), \\ \bar{N}_{l, y}^{[i]} & =\sum_{e \in S_{i}}\left(w_{e} N_{l, y}^{[i][e]}\right)\end{aligned}$

where $N_{l . x}^{[i][e]}$ is the derivative of $N_{l}^{[i]}$ computed in element $e$, and $w_{e}$ the weight function of element $e \in S_{i}$ is defined as

$w_{e}=\frac{\Delta_{e}}{\sum_{\tilde{e} \in S_{i}} \Delta_{\tilde{e}}}$, with $e \in S_{i}$

and $\Delta_{e}$ representing the area of the element $e$.

In Eq. (18), functions $\phi_{i}, \phi_{i x}$, and $\phi_{i y}$ forming the polynomial basis associated with node $i$ must satisfy the following conditions ${ }^{1}$ :

$\phi_{i}\left(\mathbf{x}_{l}\right)=\delta_{i l}, \phi_{i, x}\left(\mathbf{x}_{l}\right)=0, \phi_{i, y}\left(\mathbf{x}_{l}\right)=0$,
$\phi_{i x}\left(\mathbf{x}_{l}\right)=0, \phi_{i x, x}\left(\mathbf{x}_{l}\right)=\delta_{i l}, \phi_{i x, y}\left(\mathbf{x}_{l}\right)=0$,
$\phi_{i y}\left(\mathbf{x}_{l}\right)=0, \phi_{i y, x}\left(\mathbf{x}_{l}\right)=0, \phi_{i y, y}\left(\mathbf{x}_{l}\right)=\delta_{i l}$,

where $l$ is any one of the indices $i, j, k$, and $m$, and

$\delta_{i l}=\left\{\begin{array}{lll}1 & \text { if } & i=l \\ 0 & \text { if } & i \neq l\end{array}\right.$

We note that restrictions analogous to those listed in Eq. (18) apply to other functions, i.e., $\phi_{j}, \phi_{j x}, \phi_{j y}, \phi_{k}, \phi_{k x}, \phi_{k y}$ and $\phi_{m}, \phi_{m x}$ $\phi_{m y}$. The polynomial basis functions $\phi_{i}, \phi_{i x}$ and $\phi_{i y}$ for the CQ4 element are given by

$$
\begin{aligned}
\phi_{i}= & L_{i}+L_{i}^{2} L_{j}+L_{i}^{2} L_{k}+L_{i}^{2} L_{m}-L_{i} L_{j}^{2}-L_{i} L_{k}^{2}-L_{i} L_{m}^{2}, \\
\phi_{i x}= & -\left(x_{i}-x_{j}\right)\left(L_{i}^{2} L_{j}+p L_{i} L_{j} L_{k}+p L_{i} L_{j} L_{m}\right)-\left(x_{i}-x_{k}\right)\left(L_{i}^{2} L_{k}\right. \\
& \left.+p L_{i} L_{k} L_{m}+p L_{i} L_{k} L_{j}\right)-\left(x_{i}-x_{m}\right)\left(L_{i}^{2} L_{m}+p L_{i} L_{m} L_{j}\right. \\
& \left.+p L_{i} L_{m} L_{k}\right), \\
\phi_{i y}= & -\left(y_{i}-y_{j}\right)\left(L_{i}^{2} L_{j}+p L_{i} L_{j} L_{k}+p L_{i} L_{j} L_{m}\right)-\left(y_{i}-y_{k}\right)\left(L_{i}^{2} L_{k}\right. \\
& \left.+p L_{i} L_{k} L_{m}+p L_{i} L_{k} L_{j}\right)-\left(y_{i}-y_{m}\right)\left(L_{i}^{2} L_{m}+p L_{i} L_{m} L_{j}\right. \\
& \left.+p L_{i} L_{m} L_{k}\right) .
\end{aligned}
$$

In Eq. (23), $p=1 / 2$, and $L_{i}, L_{j}, L_{k}$ and $L_{m}$ are area coordinates of the point $\mathbf{x}$ in the quadrilateral element. Functions $\phi_{j}, \phi_{j x}, \phi_{j y} ; \phi_{k}, \phi_{k x}, \phi_{k y}$ and $\phi_{m}, \phi_{m x}, \phi_{m y}$ are found by cyclic permutation of indices $i, j, k$ and $m$.

We note that the CQ4 shape functions are complete polynomials, satisfy properties of the partition of unity, and possess the Kronecker delta property. They are not rational polynomials, and

$\tilde{N}_{l}=\underbrace{\phi_{i} N_{l}^{[i]}+\phi_{i x} \bar{N}_{l, x}^{[i]}+\phi_{i y} \bar{N}_{l, y}^{[i]}}_{\text {node } i}+\underbrace{\phi_{j} N_{l}^{[j]}+\phi_{j x} \bar{N}_{l, x}^{[j]}+\phi_{j y} \bar{N}_{l, y}^{[j]}}_{\text {node } j}+\underbrace{\phi_{k} N_{l}^{[k]}+\phi_{k x} \bar{N}_{l, x}^{[k]}+\phi_{k y} \bar{N}_{l, y}^{[k]}}_{\text {node } k}+\underbrace{\phi_{m} N_{l}^{[m]}+\phi_{m x} \bar{N}_{l, x}^{[m]}+\phi_{m y} \bar{N}_{l, y}^{[m]}}_{\text {node } m}$ 


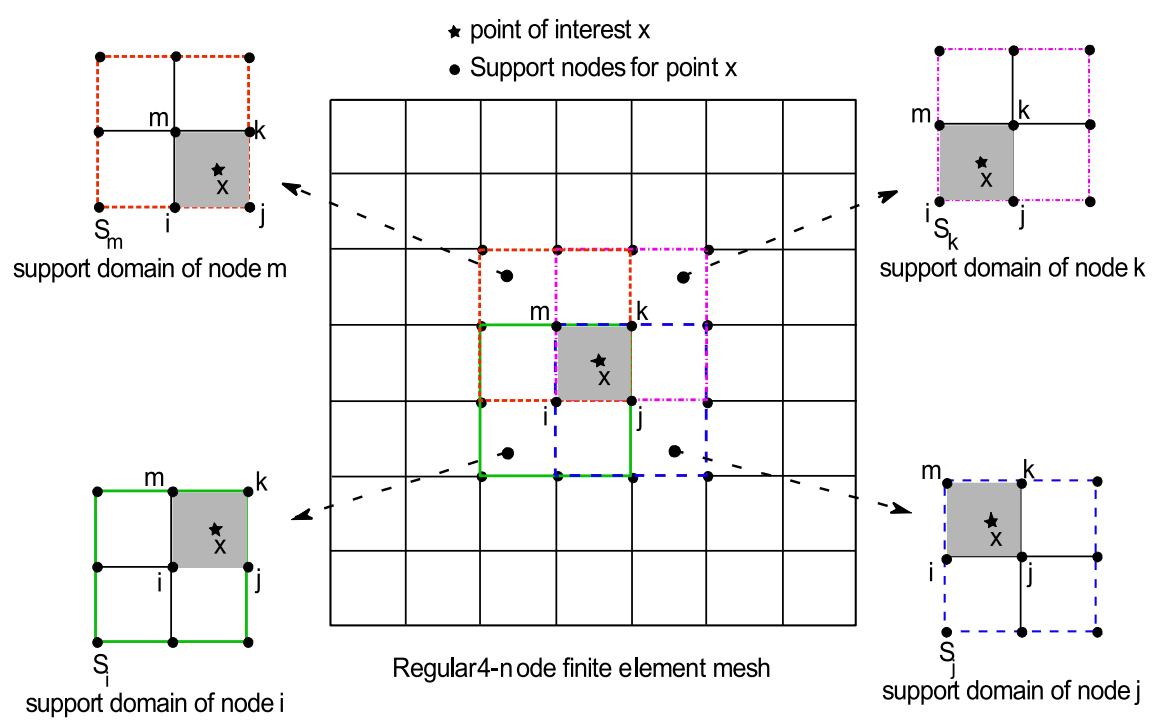

Fig. 1. Schematic representation of a consecutive-interpolation 4-node quadrilateral element and its support domains.

the stiffness and the mass matrices can be as accurately computed as those for regular 4-node quadrilateral elements. The shape functions are $C^{\infty}$ in the element interior, $C^{1}$ at nodes and $C^{0}$ on element edges.

Even though Eq. (12) for nodal displacements is similar to that for the standard quadrilateral element, the stiffness and the mass matrices for the CQ4 element differ from that of the standard Q4 element because the supporting domains of the CIP-based elements are larger than that of the Q4 element. This implies that displacements of point $\mathbf{x}$ depend upon displacements of nodes other than those at the corners of the element containing the point $\mathbf{x}$. Therefore, additional work is needed to search for supporting nodes that is not necessary in the traditional FEM.

When numerically evaluating the element mass and stiffness matrices, the number of integration points needed to exactly evaluate the integrals depends upon the degree of the highest order monomial in the integrand provided that it is a polynomial. When the integrand is not a polynomial, the integration rule is determined iteratively. Here we adopt the $3 \times 3$ Gaussian rule.

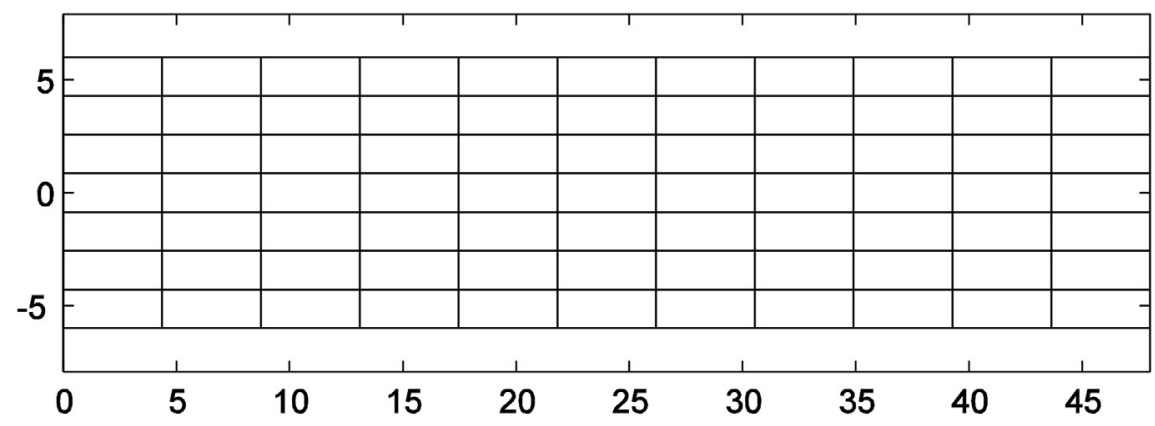

(a)

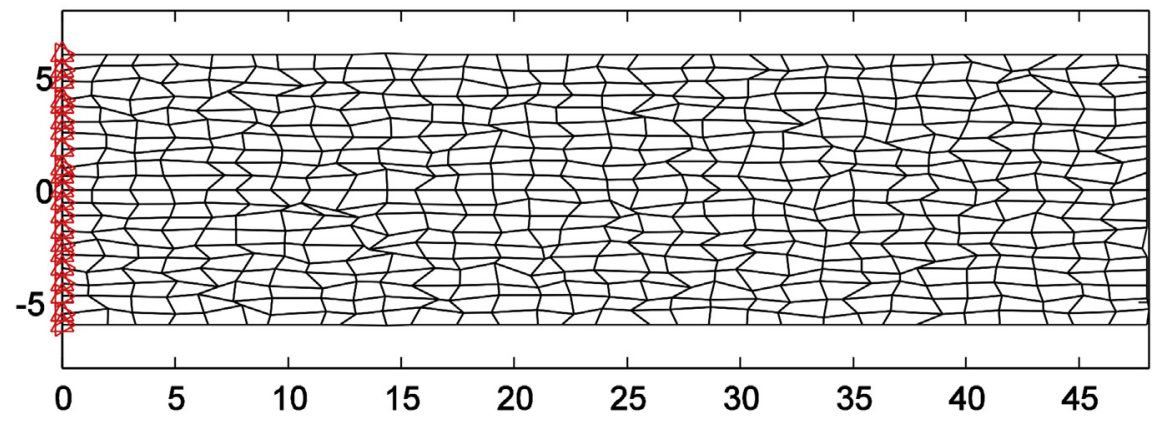

(b)

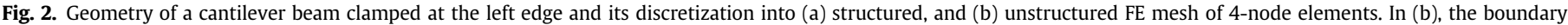

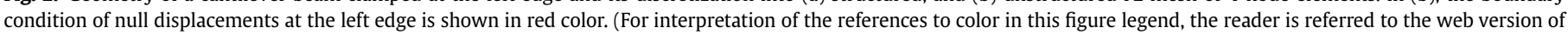
this article.). 


\section{Free vibrations}

When studying free vibrations, we set the external force equal to zero, and assume that $\mathbf{d}=\overline{\mathbf{d}} \exp (i \omega t)$ where $i=\sqrt{ }-1, \overline{\mathbf{d}}$ is the eigenvector associated with the natural or the eigen-frequency $\omega$. These are solutions of

$\left(-\omega^{2} \mathbf{M}+\mathbf{K}\right) \overline{\mathbf{d}}=0$

We present below results for several problems from simple to complex geometries and compare the presently computed frequencies and mode shapes with those reported in the literature. For a few problems we also compare solutions obtained by using the conventional three-node triangular (T3) and the four-node quadrilateral element (Q4), and the CIP-based three-node triangular element (CT3) (Zheng et al., 2010).

\subsection{Cantilever beam}

We study free vibration of a cantilever beam clamped at the left edge as shown in Fig. 2, and in consistent units, assign the following values to its material and geometric parameters: length $L=48$, thickness $D=12$, width $\widehat{t}=1.0$, Young's modulus $E=3.0 \times 10^{7}$, Poisson's ratio $\nu=0.3$, and mass density $\rho=1.0$. A structured and a non-structured discretization of the domain occupied by the undeformed beam are depicted in Fig. 2, and the first six eigenmodes
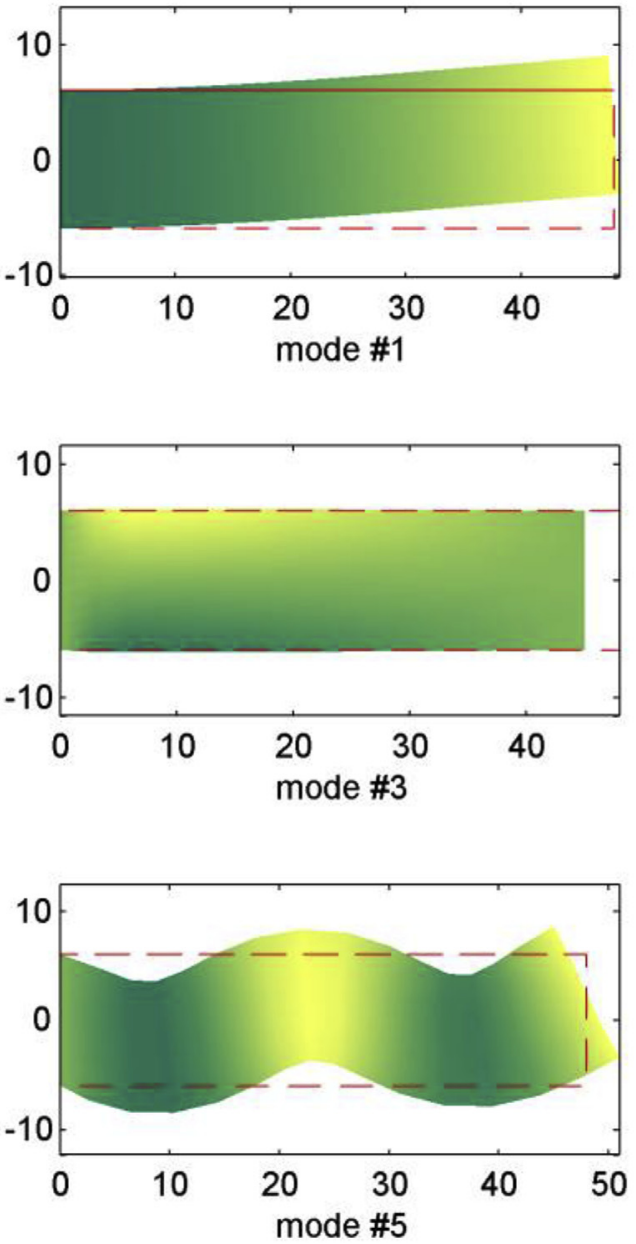

of the beam computed using the CQ4 element are displayed in Fig. 3. We have magnified displacements by a factor of 40 to easily visualize the mode shapes.

We have listed in Table 1 frequencies obtained by using the CQ4, the conventional Q4, and the CT3 elements and their \% deviations from values reported in Li et al. (2004) computed by the FEM using 4850 degrees of freedom (DoFs). The structured mesh had $30 \times 20$ uniform elements. From the results reported in Table 1, we conclude that the present approach gives very good values of the frequencies with the maximum deviation from those reported in $\mathrm{Li}$ et al. (2004) of less than $0.2 \%$. Frequencies found using the conventional Q4 element are less accurate than those computed with the CQ4 element. The convergence of the six frequencies with an increase in the number of elements in the structured and the unstructured FE meshes using CQ4 elements is reported in Tables 2 and 3 and the results are also plotted in Fig. 4. We note that as in the traditional FEM, the frequencies first converge monotonically from above as the FE mesh is refined. However, at the last stage of the FE mesh refinement, frequencies for the finer mesh are a little higher than those for the immediately previous FE mesh. Whereas the coarse mesh of $11 \times 7$ elements gives acceptable values of the first six frequencies, the $5 \times 5 \mathrm{FE}$ mesh results in rather large errors in frequencies of higher modes of vibration. Furthermore, the effect of the number of Gauss quadrature points on the frequencies has been analyzed and the results using a structured FE mesh of $21 \times 16$ uniform elements exhibited in Fig. 5 suggest that at least $2 \times 2$
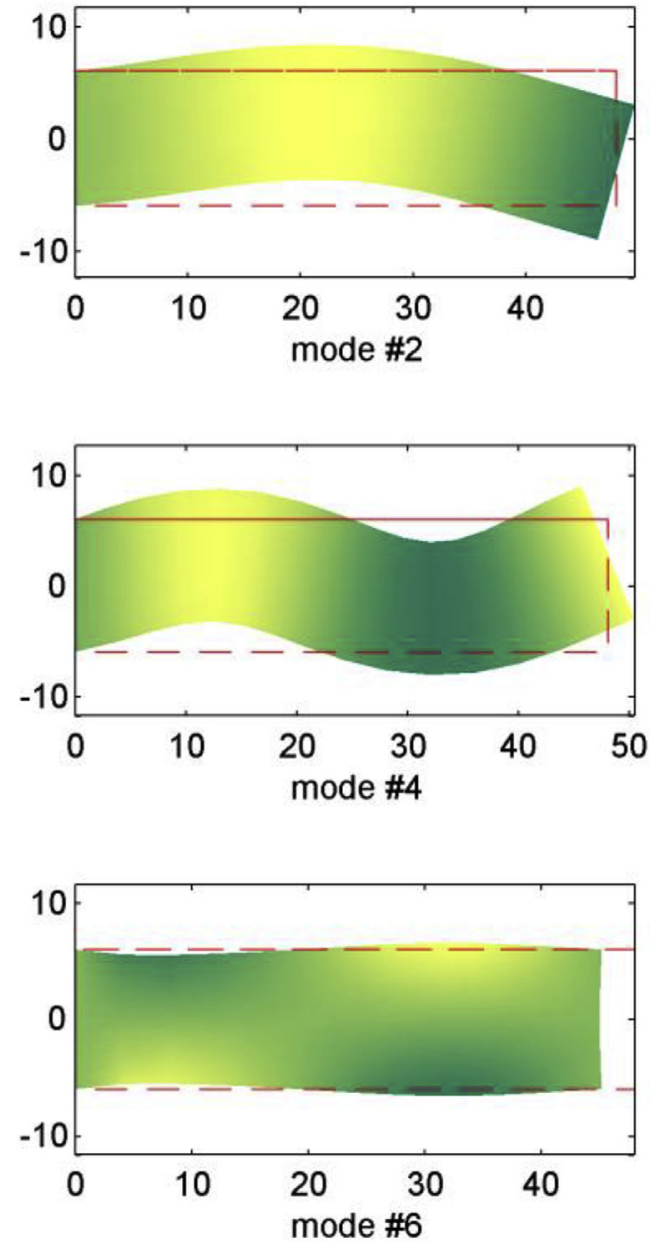

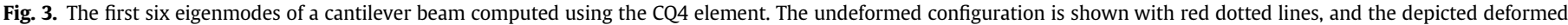

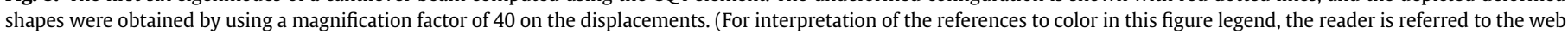
version of this article.) 
Table 1

Comparison of the first six natural frequencies $(\mathrm{Hz})$ of a cantilever beam obtained by different methods.

\begin{tabular}{|c|c|c|c|c|c|c|c|}
\hline Mode & FEM $^{\mathrm{a}} 4850$ DoFs & LoKriging $^{\mathrm{b}}$ & $\mathrm{MWS}^{\mathrm{c}}$ & $\mathrm{MK}^{\mathrm{d}}$ & CT3 & Q4 & CQ4 \\
\hline $\begin{array}{l}\omega_{1} \\
\text { Error (\%) }\end{array}$ & $\begin{array}{l}27.72 \\
-\end{array}$ & $\begin{array}{l}27.76 \\
(0.144)\end{array}$ & $\begin{array}{l}27.84 \\
(0.422)\end{array}$ & $\begin{array}{l}27.78 \\
(0.220)\end{array}$ & $\begin{array}{l}27.77 \\
(0.165)\end{array}$ & $\begin{array}{l}27.43 \\
(1.046)\end{array}$ & $\begin{array}{l}27.76 \\
(0.158)\end{array}$ \\
\hline $\begin{array}{l}\omega_{2} \\
\text { Error (\%) }\end{array}$ & $\begin{array}{l}140.86 \\
-\end{array}$ & $\begin{array}{l}140.46 \\
(0.283)\end{array}$ & $\begin{array}{l}141.13 \\
(0.191)\end{array}$ & $\begin{array}{l}142.53 \\
(1.182)\end{array}$ & $\begin{array}{l}141.07 \\
(0.148)\end{array}$ & $\begin{array}{l}145.09 \\
(3.003)\end{array}$ & $\begin{array}{l}141.07 \\
(0.149)\end{array}$ \\
\hline $\begin{array}{l}\omega_{3} \\
\text { Error (\%) }\end{array}$ & $\begin{array}{l}179.71 \\
-\end{array}$ & $\begin{array}{l}178.81 \\
(0.500)\end{array}$ & $\begin{array}{l}179.91 \\
(0.109)\end{array}$ & $\begin{array}{c}179.78 \\
(0.039)\end{array}$ & $\begin{array}{l}179.76 \\
(0.025)\end{array}$ & $\begin{array}{l}186.74 \\
(3.912)\end{array}$ & $\begin{array}{l}179.77 \\
(0.030)\end{array}$ \\
\hline $\begin{array}{l}\omega_{4} \\
\text { Error (\%) }\end{array}$ & $\begin{array}{l}323.89 \\
-\end{array}$ & $\begin{array}{l}323.83 \\
(0.018)\end{array}$ & $\begin{array}{l}323.85 \\
(0.012)\end{array}$ & $\begin{array}{l}331.39 \\
(2.314)\end{array}$ & $\begin{array}{l}324.33 \\
(0.136)\end{array}$ & $\begin{array}{l}339.36 \\
(4.776)\end{array}$ & $\begin{array}{l}324.33 \\
(0.136)\end{array}$ \\
\hline $\begin{array}{l}\omega_{5} \\
\text { Error (\%) }\end{array}$ & $\begin{array}{l}523.43 \\
-\end{array}$ & $\begin{array}{l}523.96 \\
(0.101)\end{array}$ & $\begin{array}{l}522.33 \\
(0.210)\end{array}$ & $\begin{array}{l}538.61 \\
(2.899)\end{array}$ & $\begin{array}{l}524.24 \\
(0.154)\end{array}$ & $\begin{array}{l}530.11 \\
(1.276)\end{array}$ & $\begin{array}{l}524.15 \\
(0.136)\end{array}$ \\
\hline $\begin{array}{l}\omega_{6} \\
\text { Error (\%) }\end{array}$ & $\begin{array}{l}536.57 \\
-\end{array}$ & $\begin{array}{l}534.12 \\
(0.457)\end{array}$ & $\begin{array}{l}537.15 \\
(0.107)\end{array}$ & $\begin{array}{l}542.06 \\
(1.023)\end{array}$ & $\begin{array}{l}536.69 \\
(0.024)\end{array}$ & $\begin{array}{l}542.21 \\
(1.051)\end{array}$ & $\begin{array}{l}536.74 \\
(0.031)\end{array}$ \\
\hline
\end{tabular}

a Li et al. (2004).

b Li et al. (2004).

c Gu and Liu (2005).

d Bui and Nguyen (2011).

Table 2

Convergence of natural frequencies $(\mathrm{Hz})$ of a cantilever beam using different structured meshes.

\begin{tabular}{llrrrrr}
\hline \multirow{2}{*}{ Mode } & $\begin{array}{l}\text { FEM (Li et al., 2004) } \\
\text { 4850 DoFs }\end{array}$ & \multicolumn{1}{l}{ CQ4 } \\
\cline { 3 - 7 } & $5 \times 5$ & $11 \times 7$ & $15 \times 10$ & $21 \times 16$ & $30 \times 20$ \\
\hline 1 & 27.72 & 28.88 & 27.88 & 27.80 & 27.76 & 27.76 \\
2 & 140.86 & 147.94 & 141.72 & 141.25 & 141.02 & 141.07 \\
3 & 179.71 & 180.38 & 179.88 & 179.80 & 179.76 & 179.77 \\
4 & 323.89 & 357.57 & 326.44 & 324.87 & 324.17 & 324.33 \\
5 & 523.43 & 542.96 & 529.78 & 525.51 & 523.85 & 524.15 \\
6 & 536.57 & 636.90 & 537.34 & 536.93 & 536.71 & 536.74 \\
\hline
\end{tabular}

integration rule should be used in order to accurately compute the 50 th frequency. However, in the remaining example problems studied herein, we have used the $3 \times 3$ Gauss points since several higher modes may participate in the forced transient response of the structure. We note that frequencies and mode shapes computed here need not agree with those found by using a beam theory since here we have studied plane stress deformations whereas plane strain deformations are assumed in the beam theory.

We note that the standard Q4 element may not perform well during bending deformations of a cantilever beam. In order to see if using the 8-node quadrilateral element will give better results than the CQ4 element with the same number of unknowns in the two cases, we have computed results with the commercial FE software, ANSYS, using the PLANE183 (quadratic 8-node element) and listed in Table 4 the first six natural frequencies of the beam. The two sets of results are very close to each other.

\subsection{Tapered cantilever plate with a central circular hole}

We now study free vibrations of a tapered cantilever plate with a central circular hole schematically depicted in Fig. 6 (Zhao and Steven, 1996), and in consistent units set the length $L=10$, the radius $R$ of the hole $=1.5$, Young's modulus $E=1.0$, Poisson's ratio $\nu=0.3$ and the mass density $\rho=1.0$. The asymptotic solution given in Zhao and Steven (1996) is used as the reference. The first six eigenmodes are displayed in Fig. 7 and the percentage deviations in the presently computed results with the CQ4 and the CT3 elements from those given in Zhao and Steven (1996) are summarized in Table 5. In Table 6 we have compared the first six frequencies computed with the commercial FE software ANSYS using 8-node quadratic element (PLANE183 with 1662 DoFs) and the CQ4 element (1658 DoFs). A very good agreement between both solutions is found. In the plots of Fig. 7 displacements have been multiplied by a factor of 40 . Thus deformations are really infinitesimal. It is clear that as compared to the results from the T3 and Q4 elements, frequencies computed with the CT3, the CT4 and the PLANE183 elements have significantly lower errors. Thus for this problem, the CT3 and the CT4 elements give superior results than the T3 and the Q4 elements. While computing these results we did not check if any two nodes on the boundary of the circular opening contacted each other during free vibrations of the structure. For a linear elastic problem, displacement gradients should be infinitesimal. However, for a linear elastic problem involving cracks the displacement gradients approach infinity at a crack-tip. Similarly stress and strain concentrations may occur at some points on the surface of the circular hole.

Table 3

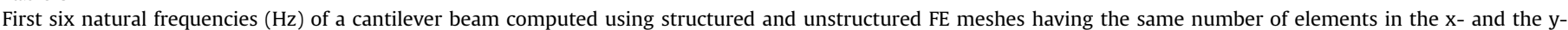
directions.

\begin{tabular}{|c|c|c|c|c|c|c|c|c|}
\hline \multirow[t]{3}{*}{ Mode } & \multirow[t]{3}{*}{ FEM $^{\mathrm{a}} 4850$ DoFs } & \multirow[t]{3}{*}{ Q4 $30 \times 20$ Struct. } & \multicolumn{4}{|l|}{ CQ4 } & \multirow{2}{*}{\multicolumn{2}{|c|}{$\frac{\text { CT3 }}{30 \times 20}$}} \\
\hline & & & \multicolumn{2}{|l|}{$15 \times 10$} & \multicolumn{2}{|l|}{$30 \times 20$} & & \\
\hline & & & Unstruc. & Struct. & Unstruc. & Struc. & Unstruc. & Struc. \\
\hline 1 & 27.72 & 27.43 & 28.08 & 27.80 & 27.77 & 27.76 & 27.85 & 27.77 \\
\hline 2 & 140.86 & 145.09 & 142.80 & 141.25 & 141.30 & 141.07 & 141.61 & 141.07 \\
\hline 3 & 179.71 & 186.74 & 180.12 & 179.80 & 179.71 & 179.77 & 179.66 & 179.76 \\
\hline 4 & 323.89 & 339.36 & 329.86 & 324.87 & 324.90 & 324.33 & 326.20 & 324.33 \\
\hline 5 & 523.43 & 530.11 & 534.62 & 525.51 & 526.02 & 524.15 & 528.89 & 524.24 \\
\hline 6 & 536.57 & 542.21 & 538.23 & 536.93 & 536.92 & 536.74 & 536.69 & 536.69 \\
\hline
\end{tabular}

${ }^{\text {a }}$ Li et al. (2004). 


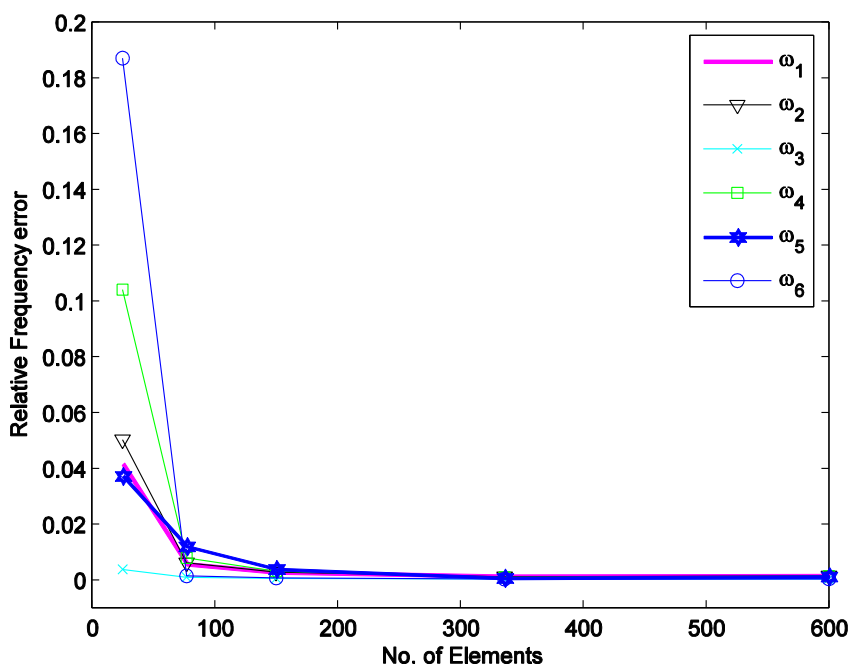

Fig. 4. With an increase in the number of elements in the FE mesh, deviation from that reported in Li et al. (2004) of the presently computed first six frequencies obtained by using the structured $5 \times 5,11 \times 7,15 \times 10,21 \times 16$ and $30 \times 20$ FE meshes using the CQ4 uniform element.

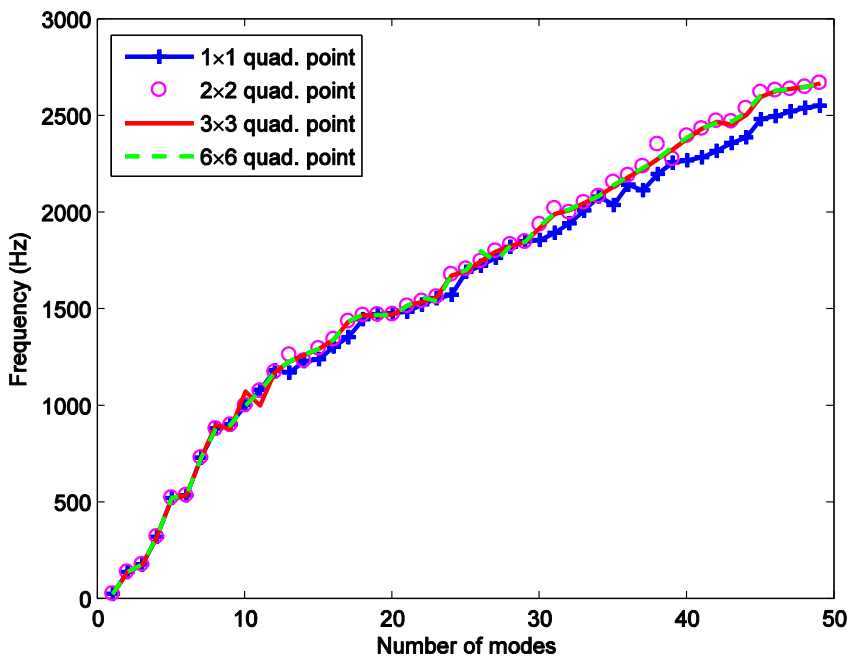

Fig. 5. Effect of the number of Gauss quadrature points on the first 50 frequencies of a cantilever beam computed using a structured mesh of $21 \times 16$ CQ4 elements.

\subsection{Shear wall with four square openings}

We now illustrate the applicability of the present formulation to the analysis of free vibrations of a shear wall with four square openings illustrated in Fig. 8. The problem is solved by arbitrarily setting Young's modulus $E=10,000 \mathrm{~N} / \mathrm{m}^{2}$, Poisson's ratio $\nu=0.2$, thickness $\widehat{t}=1.0 \mathrm{~m}$ and the mass density $\rho=1.0 \mathrm{~kg} / \mathrm{m}^{3}$. The bottom surface of the wall is clamped. The first six natural frequencies computed by using structured (476 elements) and unstructured

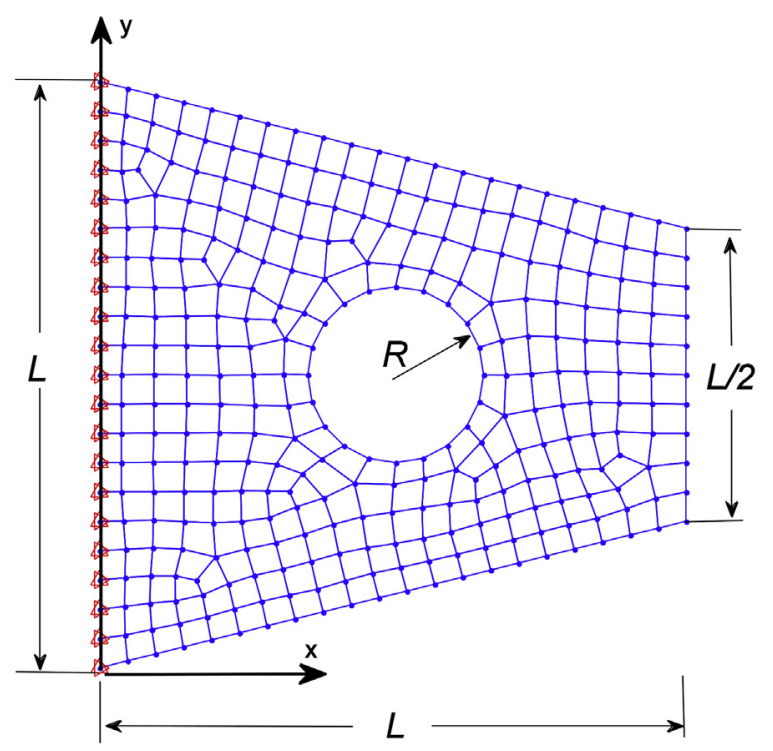

Fig. 6. Geometry of a tapered cantilever plate with a central circular hole and its discretization into an unstructured coarse mesh of 4-node quadrilateral elements.

(239 \& 476 elements) meshes are compared in Table 7 with those from the numerical solutions based on the FEM (Gu and Liu, 2001), the MLPG (Gu and Liu, 2001), the MK (Bui et al., 2011a,b), the SFEM (Dai and Liu, 2007), and the BEM (Brebbia et al., 1984). The corresponding eigenmodes of a shear wall are depicted in Fig. 9. Results reported in Table 7 indicate that the present formulation using the CQ4 element gives very good values of the first six natural frequencies. As for the above two example problems, frequencies computed with the CQ4 (4148 DoFs) element agree well with those calculated with the quadratic 8-node PLANE183 element (4160 DoFs) in ANSYS; the two sets of results are listed in Table 8.

\subsection{Car door}

We now study free vibrations of a car door that have been studied in Cui et al. (2010) using a mesh-free method. The detailed configuration of the door is given in Cui et al. (2010), and a typical unstructured mesh using 609 CQ4 elements is shown in Fig. 10. The following values of geometric and material parameters are used: Young's modulus $E=210 \mathrm{MPa}$, Poisson's ratio $\nu=0.3$, mass density $\rho=7.8 \times 10^{-6} \mathrm{~kg} / \mathrm{mm}^{3}$, the thickness of the door $\widehat{t}=1.0 \mathrm{~mm}$. The first 12 natural frequencies computed using Q4, CQ4 (4872 DoFs) and T3, CT3 (4660 DoFs) elements are listed in Table 9 along with the reference solutions that used the CPS6M and SC3-L elements (Cui et al., 2010). The first six eigenmodes are shown in Fig. 11. It is clear that the present results computed by using the CQ4 and the CT3 elements agree well with those of Cui et al. (2010). For the same number of DoFs, frequencies found by using the T3 and the Q4 elements noticeably deviate from those computed by using other elements.

Table 4

First six natural frequencies (Hz) of a cantilever beam computed using ANSYS (PLANE183 8-node element) and CQ4.

\begin{tabular}{lccccrr}
\hline Mode & PLANE183 (464 DoFs) & CQ4 (462 DoFs) & PLANE183 (812 DoFs) & CQ4 (810 DoFs) & PLANE183 (1256 DoFs) & CQ4 (1254 DoFs) \\
\hline 1 & 27.74 & 27.76 & 27.73 & 27.74 & 27.72 & 27.73 \\
2 & 140.95 & 141.07 & 140.88 & 140.95 & 140.85 & 140.89 \\
3 & 179.75 & 179.76 & 179.72 & 179.74 & 179.72 & 323.74 \\
4 & 324.02 & 324.33 & 323.83 & 323.99 & 522.99 & 523.72 \\
5 & 523.57 & 524.15 & 523.15 & 523.45 & 536.54 & 53.18 \\
6 & 536.62 & 536.74 & 536.57 & 536.64 & 5.59 \\
\hline
\end{tabular}



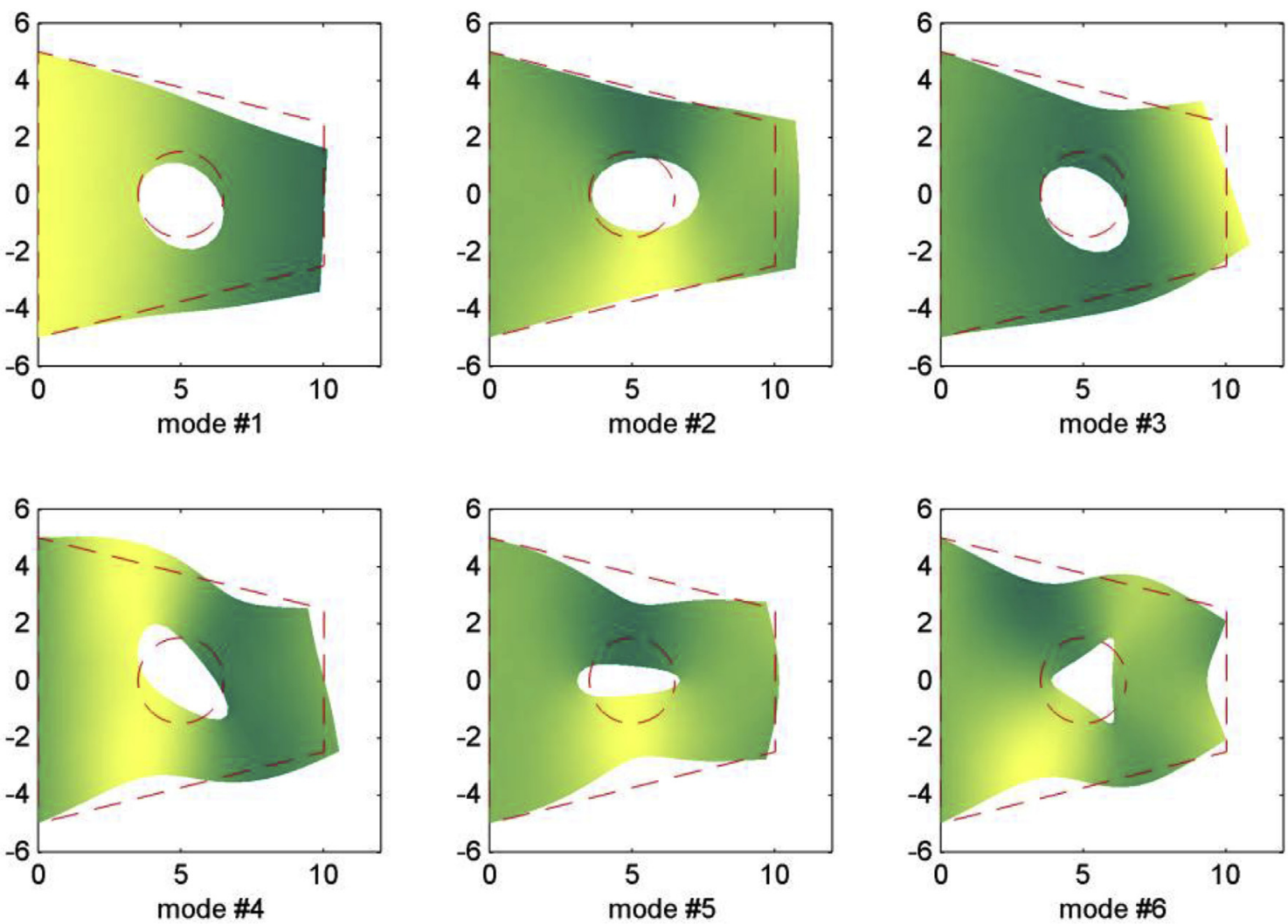

Fig. 7. First six eigenmodes of a tapered cantilever plate with a central circular hole. Displacements have been magnified by a factor of 40 in these plots.

Table 5

Comparison of natural frequencies ( $\mathrm{rad} / \mathrm{s}$ ) and the percentage error for the first six eigenmodes of a tapered cantilever plate with a central circular hole obtained by several methods.

\begin{tabular}{|c|c|c|c|c|c|c|}
\hline Mode & $\begin{array}{l}\text { Asymptotic analysis } \\
\text { (Zhao and Steven, } \\
\text { 1996) }\end{array}$ & $\begin{array}{l}\text { FEM (Zhao and } \\
\text { Steven, 1996) }\end{array}$ & T3 & CT3 & Q4 & CQ4 \\
\hline $\begin{array}{l}\omega_{1} \\
\text { Error (\%) }\end{array}$ & $\begin{array}{l}0.0744 \\
-\end{array}$ & $\begin{array}{l}0.0833 \\
(11.96)\end{array}$ & $\begin{array}{l}0.0875 \\
(17.61)\end{array}$ & $\begin{array}{l}0.0748 \\
(0.54)\end{array}$ & $\begin{array}{l}0.0774 \\
(4.03)\end{array}$ & $\begin{array}{l}0.0740 \\
(0.54)\end{array}$ \\
\hline $\begin{array}{l}\omega_{2} \\
\text { Error (\%) }\end{array}$ & $\begin{array}{l}0.1648 \\
-\end{array}$ & $\begin{array}{l}0.1661 \\
(0.79)\end{array}$ & $\begin{array}{l}0.1560 \\
(5.34)\end{array}$ & $\begin{array}{l}0.1715 \\
(4.07)\end{array}$ & $\begin{array}{l}0.1748 \\
(6.07)\end{array}$ & $\begin{array}{l}0.1652 \\
(0.24)\end{array}$ \\
\hline $\begin{array}{l}\omega_{3} \\
\text { Error (\%) }\end{array}$ & $\begin{array}{l}0.2055 \\
-\end{array}$ & $\begin{array}{l}0.2334 \\
(13.58)\end{array}$ & $\begin{array}{l}0.2499 \\
(21.61)\end{array}$ & $\begin{array}{l}0.2189 \\
(6.52)\end{array}$ & $\begin{array}{l}0.2343 \\
(14.01)\end{array}$ & $\begin{array}{l}0.2114 \\
(2.87)\end{array}$ \\
\hline $\begin{array}{l}\omega_{4} \\
\text { Error (\%) }\end{array}$ & $\begin{array}{l}0.2939 \\
-\end{array}$ & $\begin{array}{l}0.3302 \\
(12.35)\end{array}$ & $\begin{array}{l}0.3426 \\
(16.57)\end{array}$ & $\begin{array}{l}0.2897 \\
(1.43)\end{array}$ & $\begin{array}{l}0.3155 \\
(7.35)\end{array}$ & $\begin{array}{l}0.2804 \\
(4.59)\end{array}$ \\
\hline $\begin{array}{l}\omega_{5} \\
\text { Error (\%) }\end{array}$ & $\begin{array}{l}0.3243 \\
-\end{array}$ & $\begin{array}{l}0.4234 \\
(30.56)\end{array}$ & $\begin{array}{l}0.3923 \\
(20.97)\end{array}$ & $\begin{array}{l}0.3262 \\
(0.59)\end{array}$ & $\begin{array}{l}0.3467 \\
(6.91)\end{array}$ & $\begin{array}{l}0.3210 \\
(1.02)\end{array}$ \\
\hline $\begin{array}{l}\omega_{6} \\
\text { Error (\%) }\end{array}$ & $\begin{array}{l}0.4399 \\
-\end{array}$ & $\begin{array}{l}0.4624 \\
(5.12)\end{array}$ & $\begin{array}{l}0.4901 \\
(11.41)\end{array}$ & $\begin{array}{l}0.4541 \\
(3.23)\end{array}$ & $\begin{array}{l}0.4784 \\
(8.75)\end{array}$ & $\begin{array}{l}0.4419 \\
(0.46)\end{array}$ \\
\hline
\end{tabular}

Table 6

Comparison of natural frequencies ( $\mathrm{rad} / \mathrm{s}$ ) for the first six eigenmodes of a tapered cantilever plate with a central circular hole obtained by the PLANE183 quadratic 8node element in ANSYS and the CQ4 element.

\begin{tabular}{lll}
\hline Mode & PLANE183 (ANSYS) 1662 DoFs & CQ4 1658 DoFs \\
\hline$\omega_{1}$ & 0.0700 & 0.0704 \\
$\omega_{2}$ & 0.1559 & 0.1568 \\
$\omega_{3}$ & 0.1999 & 0.2008 \\
$\omega_{4}$ & 0.2622 & 0.2619 \\
$\omega_{5}$ & 0.2919 & 0.2929 \\
$\omega_{6}$ & 0.4195 & 0.4206 \\
\hline
\end{tabular}

\subsection{A bracket}

A structure more complex than the ones studied above is a bracket schematically depicted in Fig. 12. We assign following values to the material parameters: $E=2 \times 10^{5} \mathrm{MPa}, \nu=0.3$, $\rho=7.8 \times 10^{-6} \mathrm{~kg} / \mathrm{mm}^{3}$, and the thickness of the bracket $\widehat{t}=5.0 \mathrm{~mm}$. The boundary conditions are: the periphery of the tiny circular hole at the top left side is clamped, and points on the left edge can slide in the vertical direction but have null displacements in the horizontal direction. The first six natural frequencies of the bracket with the corresponding eigenmodes displayed in Fig. 13, obtained by using the CQ4 element and an unstructured FE mesh of 1003 elements are 44.0, 95.0, 257.1, 293.9, 348.3, and $473.8 \mathrm{~Hz}$.

\section{Forced vibrations}

We use the implicit (the Newmark method) and the explicit (the central difference method (CDM)) time integration schemes to study transient deformations of a structure. We write the equation of motion, Eq. (12), at time $t+\Delta t$ as

$$
\mathbf{M} \ddot{\mathbf{d}}_{t+\Delta t}+\mathbf{C} \dot{\mathbf{d}}_{t+\Delta t}+\mathbf{K} \mathbf{d}_{t+\Delta t}=\mathbf{f}_{t+\Delta t}
$$

The Newmark time integration scheme applied to Eq. (25) gives (e.g., see Bui et al., 2013; Zienkiewicz et al., 2005)

$$
\begin{aligned}
{\left[\mathbf{M}+\beta \Delta t^{2} \mathbf{K}+\gamma \Delta t \mathbf{C}\right] \ddot{\mathbf{d}}_{t+\Delta t} } & =\mathbf{f}_{t+\Delta t}-\mathbf{K}\left[\mathbf{d}_{t}+\Delta t \dot{\mathbf{d}}_{t}+(1-2 \beta) \frac{\Delta t^{2}}{2} \ddot{\mathbf{d}}_{t}\right] \\
& -\mathbf{C}\left(\dot{\mathbf{d}}_{t}+(1-\gamma) \Delta t \ddot{\mathbf{d}}_{t}\right)
\end{aligned}
$$




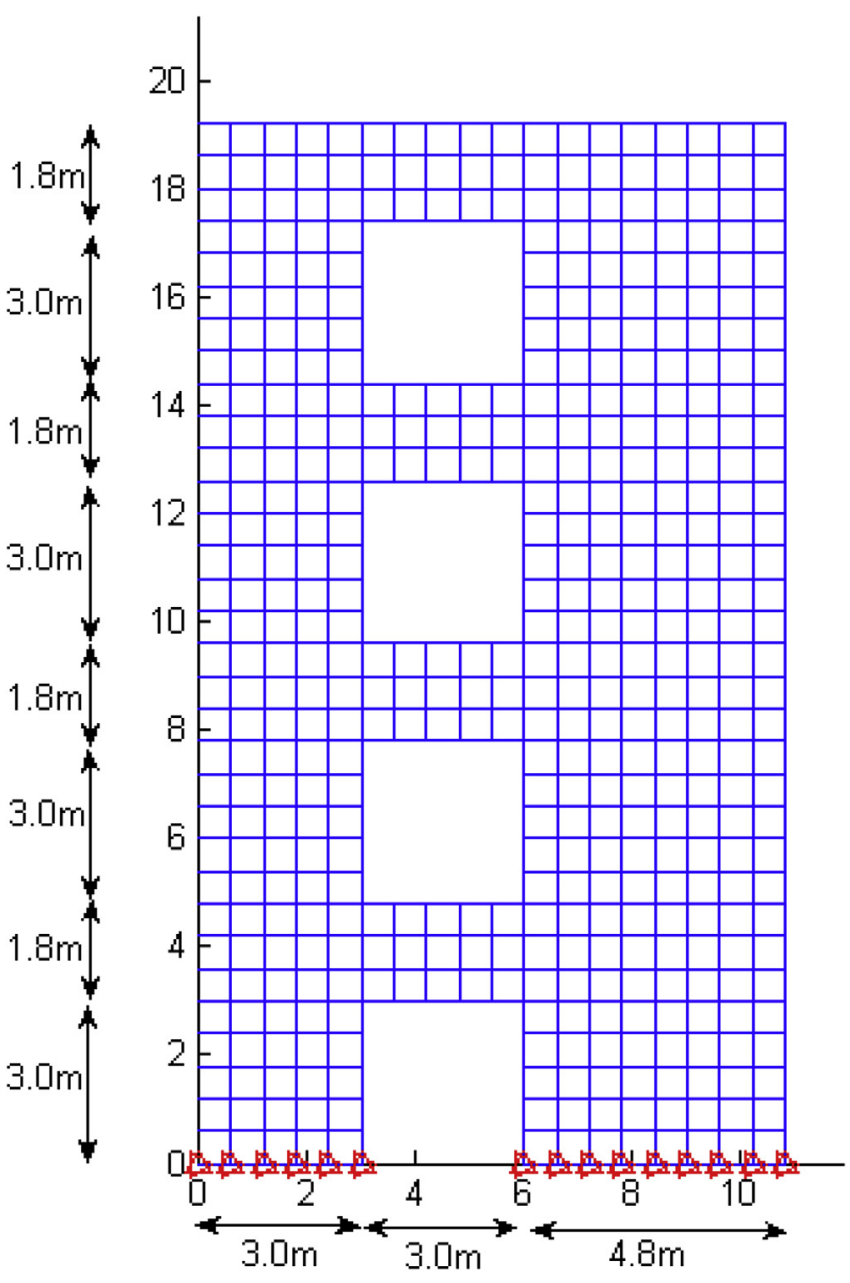

Fig. 8. Geometric parameters of a shear wall with four openings.

where $\Delta t$ is the time step. After solving Eq. (26) for $\ddot{\mathbf{d}}_{t+\Delta t}$ under the pertinent essential boundary conditions, the displacement $\mathbf{d}_{t+\Delta t}$ and the velocity $\dot{\mathbf{d}}_{t+\Delta t}$ at time $t+\Delta t$ are then found, respectively, from

$$
\begin{aligned}
& \mathbf{d}_{t+\Delta t}=\mathbf{d}_{t}+\Delta t \dot{\mathbf{d}}_{t}+(1-2 \beta) \frac{\Delta t^{2}}{2} \ddot{\mathbf{d}}_{t}+\beta \Delta t^{2} \ddot{\mathbf{d}}_{t+\Delta t} \\
& \dot{\mathbf{d}}_{t+\Delta t}=\dot{\mathbf{d}}_{t}+(1-\gamma) \Delta t \ddot{\mathbf{d}}_{t}+\gamma \Delta t \ddot{\mathbf{d}}_{t+\Delta t}
\end{aligned}
$$

The Newmark method is unconditionally stable for $\gamma \geq 0.5$ and $\beta \geq 0.25(\gamma+0.5)^{2}$, otherwise it is conditionally stable. Here we use both the unconditionally stable implicit method in which we take $\gamma=0.5$ and $\beta=0.25$, and the conditionally stable explicit CDM for which $\gamma=0.5$ and $\beta=0$ (Zienkiewicz et al., 2005; Gu and Liu, 2001).
The critical time step for computing the stable solution with the CDM is given by

$\Delta t_{c r}=\frac{L^{e}}{c_{d}}$

where $L^{e}$ is the length of the smallest element in the FE mesh, and the wave speed $c_{d}$ is found from

$c_{d}=\sqrt{\frac{E(1-\nu)}{(1+\nu)(1-2 \nu) \rho}}$

Even when $\Delta t<\Delta t_{c r}$, the accuracy of the solution for both the implicit and the explicit methods depends upon $\Delta t$.

\subsection{Forced vibration of a cantilever beam}

The first example problem studied is that of a cantilever beam, shown in Fig. 14, subjected to a parabolic traction at the right unclamped end. Three time-dependent loads, $P=1000 g(t)$ with $g(t)$ the function of time depicted in Fig. 15 are considered. The geometrical and the material parameters of the beam are the same as those for the beam studied in Section 3.1. The presently computed vertical displacement or the deflection of point A shown in Fig. 14 is compared with that obtained from the commercial FE software ANSYS (Bui et al., 2011a,b).

\subsubsection{Harmonic load}

For the loading function $g(t)=\sin 27 t$ where $t$ is in seconds, we have compared in Figs. 16 and 17 the time history of the computed vertical displacement $u_{y}$ of point A for different values of the time step $\Delta t$ and using the CQ4 and the CT3 elements with those obtained by using the commercial FE software ANSYS (Bui et al., 2011a,b). As expected, the solution accuracy increases with a decrease in the value of $\Delta t$ and the difference between the numerical solutions computed with the CQ4 and the ANSYS decreases. For the implicit method, the solution computed with $\Delta t=5 \times 10^{-2} \mathrm{~s}$ has considerably lower amplitude and larger time periods than those found with smaller values of $\Delta t$ confirming that one should compute the numerical solution for different values of $\Delta t$ even for an implicit method. In Fig. 18 we have plotted the computed time histories of $u_{y}$ for point $\mathrm{A}$ and different FE meshes. Our numerical experiments have indicated that the unstructured $\mathrm{FE}$ meshes give good results for the forced vibration analysis. The coarse $5 \times 4$ and $6 \times 6$ uniform FE meshes do not give reasonable values of the amplitude of vibration and of the time periods. However, the $20 \times 12 \mathrm{FE}$ mesh provides a solution close to the reference solution computed with ANSYS, and that found with the $20 \times 12$ uniform FE mesh. Even the $8 \times 8 \mathrm{FE}$ mesh gives good values of the deflection of point A; these results are not depicted in Fig. 18. In the remaining example problems studied here, unless otherwise noted, we have used the uniform $20 \times 12 \mathrm{FE}$ mesh.

Table 7

\begin{tabular}{|c|c|c|c|c|c|c|c|c|}
\hline \multirow[t]{2}{*}{ Mode } & \multirow{2}{*}{$\begin{array}{l}\text { BEM (Brebbia et al., } \\
\text { 1984) }\end{array}$} & \multirow{2}{*}{$\begin{array}{l}\text { FEM (Gu and Liu, } \\
\text { 2001) }\end{array}$} & \multirow{2}{*}{$\begin{array}{l}\text { MLPG (Gu and Liu, } \\
\text { 2001) }\end{array}$} & \multirow{2}{*}{$\begin{array}{l}\text { MK (Bui et al., } \\
\text { 2011a,b) }\end{array}$} & \multirow{2}{*}{$\begin{array}{l}\text { SFEM (Dai and Liu, } \\
\text { 2007) }\end{array}$} & \multicolumn{3}{|l|}{ CQ4 } \\
\hline & & & & & & $\begin{array}{l}\text { Unstruc. } \\
239 \text { ele. }\end{array}$ & $\begin{array}{l}\text { Unstruc. } \\
476 \text { ele. }\end{array}$ & $\begin{array}{l}\text { Struc. } \\
476 \text { ele. }\end{array}$ \\
\hline$\omega_{1}$ & 2.079 & 2.073 & 2.069 & 2.117 & 2.059 & 2.073 & 2.064 & 2.059 \\
\hline$\omega_{2}$ & 7.181 & 7.096 & 7.154 & 7.199 & 7.059 & 7.093 & 7.070 & 7.062 \\
\hline$\omega_{3}$ & 7.644 & 7.625 & 7.742 & 7.616 & 7.619 & 7.627 & 7.624 & 7.622 \\
\hline$\omega_{4}$ & 11.833 & 11.938 & 12.163 & 12.317 & 11.827 & 11.969 & 11.897 & 11.847 \\
\hline$\omega_{5}$ & 15.947 & 15.341 & 15.587 & 15.633 & 15.238 & 15.378 & 15.286 & 15.265 \\
\hline$\omega_{6}$ & 18.644 & 18.345 & 18.731 & 18.014 & 18.275 & 18.382 & 18.288 & 18.271 \\
\hline
\end{tabular}

Comparison of natural frequencies ( $\mathrm{rad} / \mathrm{s}$ ) for the first six modes of a shear wall with four openings obtained by several methods. 

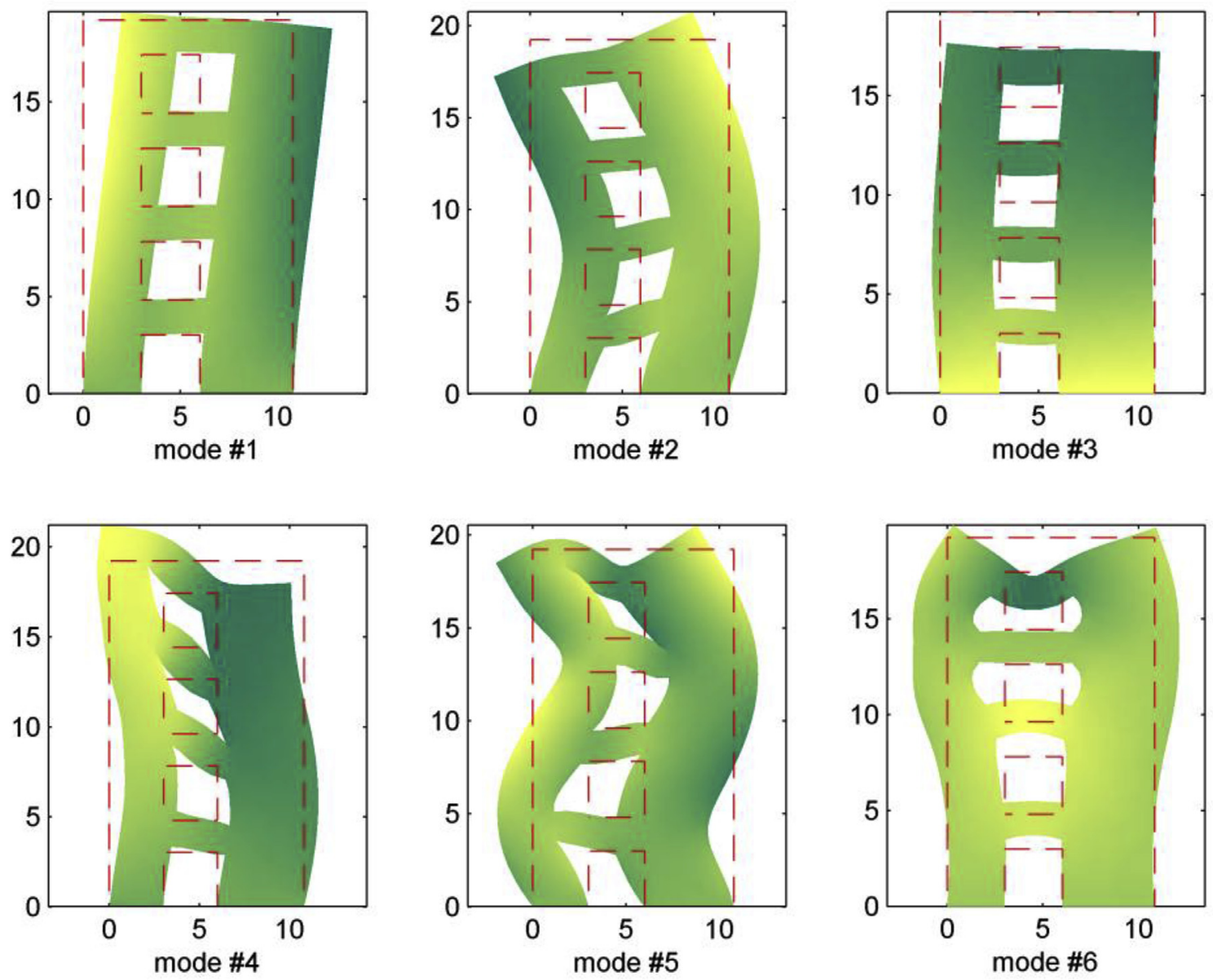

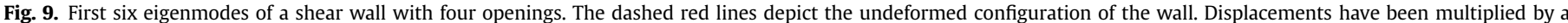

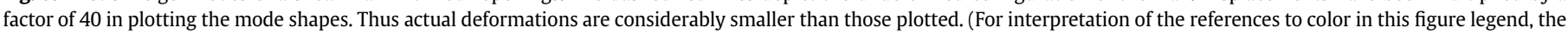
reader is referred to the web version of this article.).

The effect of the time step size has also been studied to check the stability of the integration technique. In the CDM $\Delta t=1 \times 10^{-4}$ $\mathrm{s}$ is used. The time history of the vertical deflection of point $\mathrm{A}$ computed by the CDM till $20 \mathrm{~s}$ with $(\mathrm{c}=0.4)$ and without $(\mathrm{c}=0.0)$ damping effects is plotted in Fig. 19. It is clear that the computed solution is quite stable.

\subsubsection{Load given by the Heaviside function}

For the Heaviside step load, $g(t)=H(t)$, the analytical value of the final deflection of point $\mathrm{A}$ is $u_{y \text {,exact }}=-0.0089$ units (Timoshenko and Goodier, 1970). The computed time history of the vertical deflection of point A obtained with the CDM and damping coefficient, $c=0.4$, is shown in Fig. 20. It is evident that the response

Table 8

Comparison of the first six natural frequencies ( $\mathrm{rad} / \mathrm{s}$ ) of a shear wall with four openings obtained by the PLANE183 quadratic 8-node element (ANSYS) and the CQ4 element.

\begin{tabular}{lcc}
\hline Mode & PLANE183 (ANSYS) 4160 DoFs & CQ4 4148 DoFs \\
\hline$\omega_{1}$ & 2.0189 & 2.0286 \\
$\omega_{2}$ & 6.9699 & 6.9924 \\
$\omega_{3}$ & 7.6033 & 7.6081 \\
$\omega_{4}$ & 11.5322 & 11.6083 \\
$\omega_{5}$ & 15.0181 & 15.0736 \\
$\omega_{6}$ & 18.0899 & 18.1388 \\
\hline
\end{tabular}

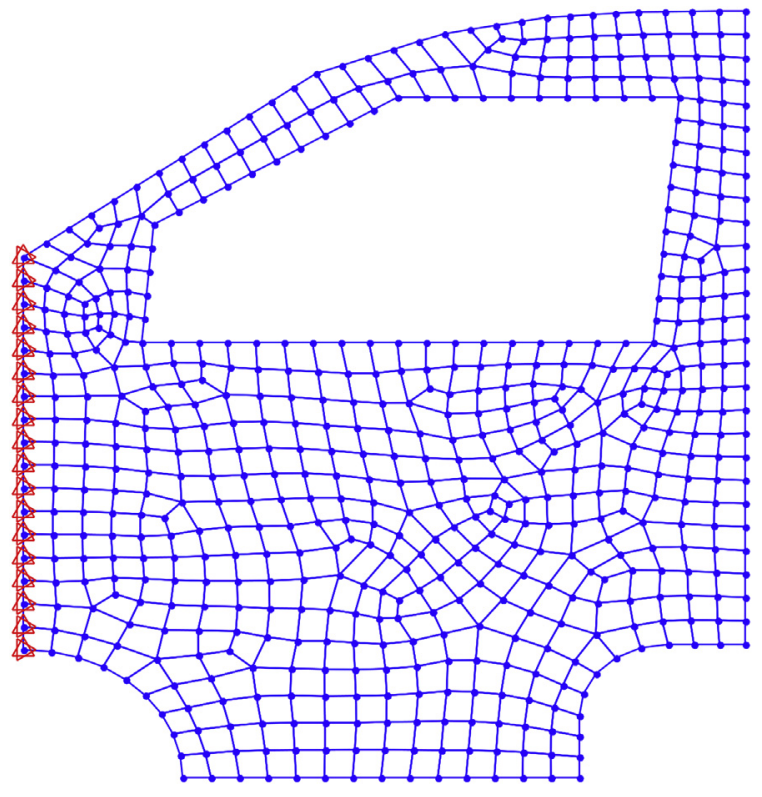

Fig. 10. An unstructured FE mesh of a car door. 

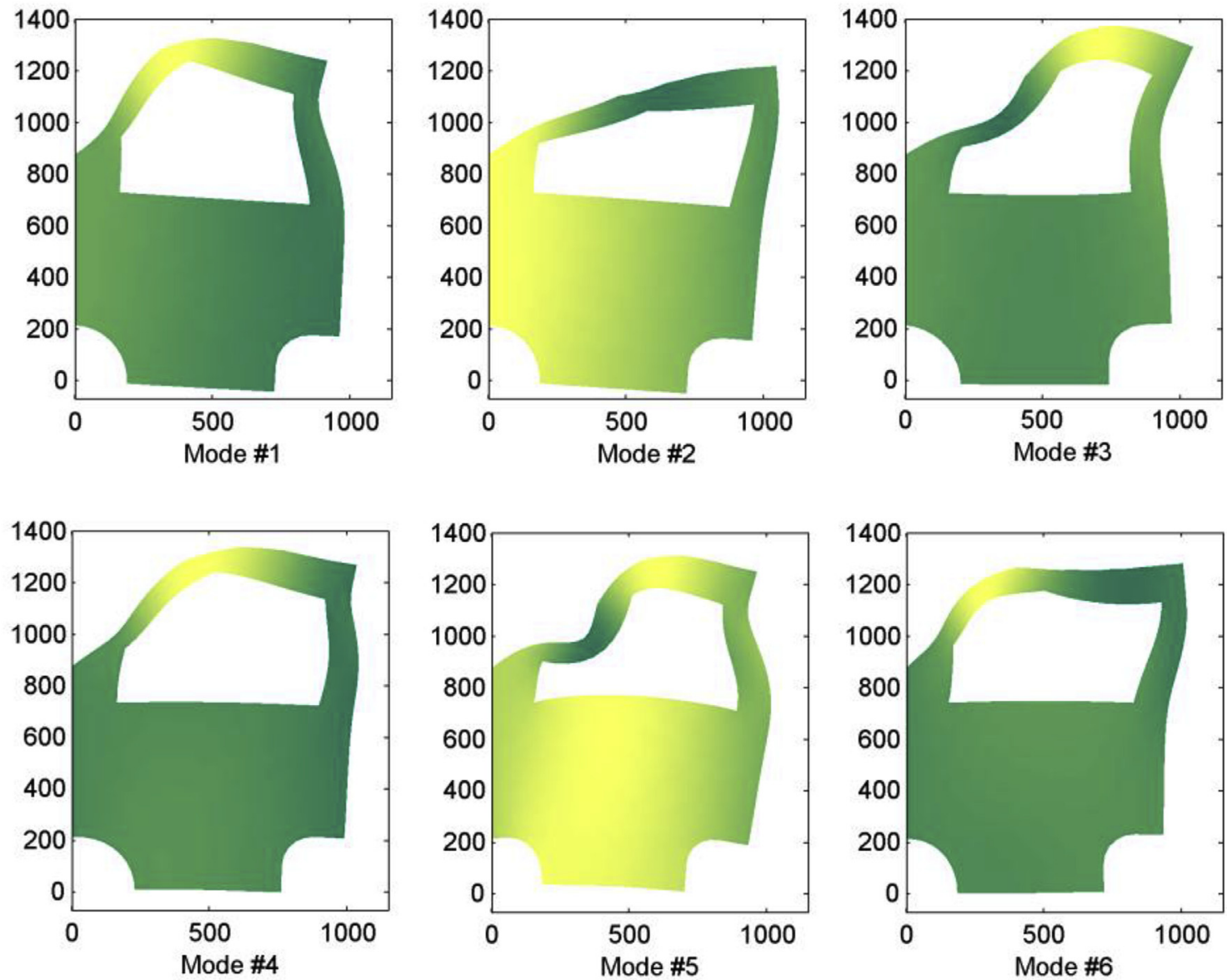

Fig. 11. First six eigenmodes of the door of a car obtained by using the CQ4 element with displacements magnified by a factor of 40 .

gradually converges to that for the statically deformed beam. This agrees well with those derived from other methods, e.g., the MK (Bui et al., 2011a,b), MWS (Gu and Liu, 2005), or LoKriging (Li et al., 2004).

For the step load given by $g(t)=\frac{t}{t_{0}} H(t)-\left(\frac{t}{t_{0}}-1\right) H\left(t-t_{0}\right)$ (Bui et al., 2011a,b), the computed time history of the vertical displacement of point A with the implicit integration method and $c=0.4$ shown in Fig. 21 agrees well with that derived from Bui et al. (2011a,b) for the MK method. When the damping is considered, the

\section{Table 9}

Comparison of the first 12 natural frequencies ( $\mathrm{rad} / \mathrm{s}$ ) of the door of a car obtained by using the CQ4, the Q4, the T3 and the CT3 elements with those from Cui et al. (2010) wherein the CPS6M and the SC3-L elements were used.

\begin{tabular}{lllllll}
\hline Mode & CPS6M & SC3-L & T3 & CT3 & Q4 & CQ4 \\
\hline 1 & 0.30205 & 0.30346 & 0.34586 & 0.30351 & 0.34239 & 0.30103 \\
2 & 0.46203 & 0.46649 & 0.76077 & 0.48279 & 0.73333 & 0.47357 \\
3 & 1.0404 & 1.0430 & 1.0315 & 1.0691 & 0.9659 & 1.0646 \\
4 & 1.2104 & 1.2177 & 1.4539 & 1.2019 & 1.4425 & 1.2117 \\
5 & 1.2814 & 1.2857 & 1.8311 & 1.3032 & 1.7456 & 1.2964 \\
6 & 1.4866 & 1.4929 & 2.1983 & 1.5180 & 2.1578 & 1.5073 \\
7 & 1.7103 & 1.7341 & 2.6499 & 1.7311 & 2.5690 & 1.7287 \\
8 & 1.8597 & 1.8656 & 2.8842 & 1.9018 & 2.8325 & 1.8913 \\
9 & 2.3729 & 2.3912 & 3.0705 & 2.4512 & 3.0454 & 2.4057 \\
10 & 2.8152 & 2.8278 & 3.5783 & 2.9039 & 3.4965 & 2.8853 \\
11 & 3.0632 & 3.0704 & 3.9157 & 3.0873 & 3.8399 & 3.0833 \\
12 & 3.3244 & 3.3349 & 3.9729 & 3.3736 & 3.9208 & 3.3676 \\
\hline
\end{tabular}

amplitude of the deflection of point A gradually decreases and converges to a steady state value.

\subsubsection{Decreasing transient load of finite duration}

For the loading function $g(t)=(1-t) H(1-t)$, the load gradually decreases from a finite value to zero in $1 \mathrm{~s}$, and then stays at zero. The time histories of the deflection $\mathrm{u}_{\mathrm{y}}$ of point $\mathrm{A}$ with and without damping are provided in Figs. 22 and 23, respectively. The computed responses with the implicit integration scheme and the CQ4 and the CT3 elements are close to each other. As expected, the amplitude of oscillations remains nearly constant for $\mathrm{c}=0$ after $1 \mathrm{~s}$ when the applied load has become zero, but approaches a steady state value for $\mathrm{c}=0.4$.

\subsection{Forced vibration of an L-shape panel}

We now analyze transient deformations of an L-shape panel, sketched in Fig. 24, and assign following values to its material and geometric parameters, and the applied load: height $H=2.0$, thickness $\widehat{t}=1.0$, Young's modulus $E=1.0$, Poisson's ratio $\nu=0.3$, mass density $\rho=1.0$, uniform pressure $P=10 \times g(t)$ where $g(t)$ is the function plotted in Fig. 15c. The panel is discretized into an unstructured FE mesh of 296 CQ4 elements. Time histories of the vertical displacement $\mathrm{u}_{\mathrm{y}}$ of point $\mathrm{A}$ computed with the explicit and the implicit methods using different values of the time step size $\Delta t$ are plotted in Fig. 25. Without damping effect, values of $\mathrm{u}_{\mathrm{y}}$ for point A oscillate around the mean value zero. 


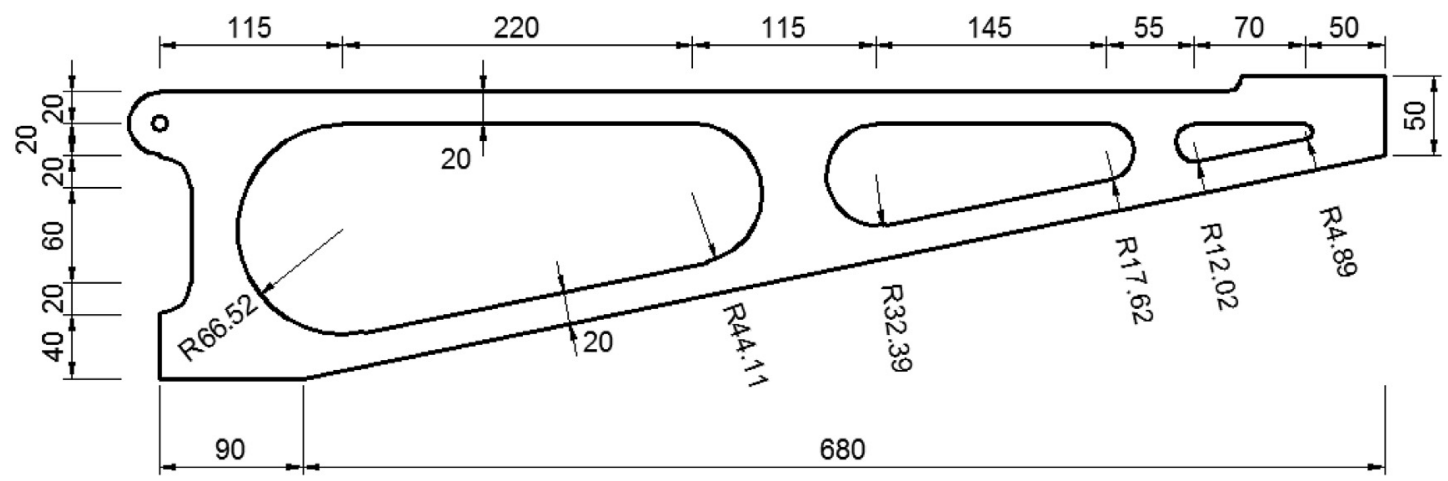

Fig. 12. Schematic sketch and geometric parameters (in $\mathrm{mm}$ ) of the bracket studied.

\section{Free vibrations of piezoelectric structures}

Piezoelectric materials, due to their ability to convert electrical into mechanical energy and vice-versa, have been widely used in many engineering applications. Recently, meshless and extended FE approaches have been developed to analyze 2D structural and fracture problems for piezoelectric solids under static, dynamic and thermal loads (Bui and Zhang, 2012, 2013; Liu et al., 2014; Allik and Hughes, 1970; Benjeddou, 2000; Sharma et al., 2013). Three-dimensional transient nonlinear problems for piezoelectric solids have been studied by Batra and Liang (1997, 1996). Threedimensional free vibrations of a piezoelectric body have been analyzed by Yang and Batra (1994). The piezoelectric effect has been exploited by Batra and Geng $(2001,2002)$ to enhance the buckling load. Here, we show that the use of the CQ4 element
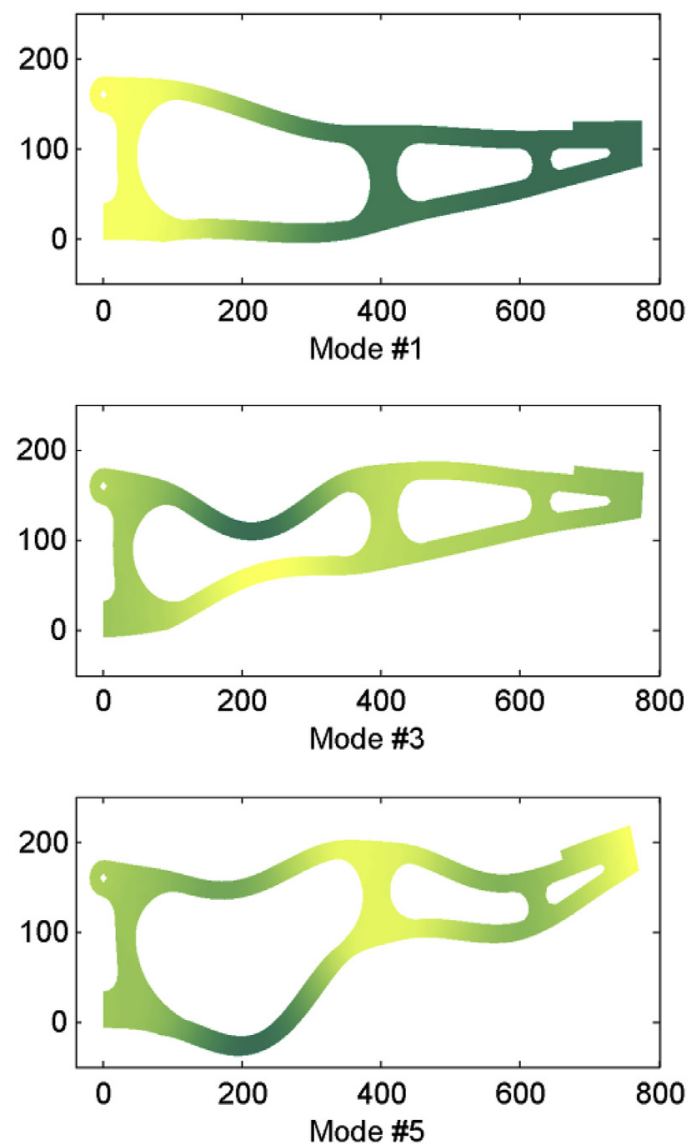

provides very good solution of the eigenvalue problem for 2-D deformations of piezoelectric structures. For the sake of completeness we briefly list below the pertinent equations.

The constitutive relation for 2-D deformations of a linear piezoelectric material is

$\left[\begin{array}{l}\boldsymbol{\sigma} \\ \mathbf{D}\end{array}\right]=\left[\begin{array}{cc}\mathbf{c}_{E} & -\mathbf{e}^{\mathrm{T}} \\ \mathbf{e} & \boldsymbol{\kappa}_{S}\end{array}\right]\left[\begin{array}{l}\boldsymbol{\varepsilon} \\ \mathbf{E}\end{array}\right]$

where $\mathbf{D}$ denotes the electric displacement, $\mathbf{E}$ the electric field, $\mathbf{c}_{E}$ the elastic matrix measured at constant electric field, e the piezoelectric matrix, and $\kappa_{S}$ the dielectric matrix at constant mechanical strain. A piezoelectric material is usually modeled as transversely isotropic with the axis of polarization coincident with the axis of
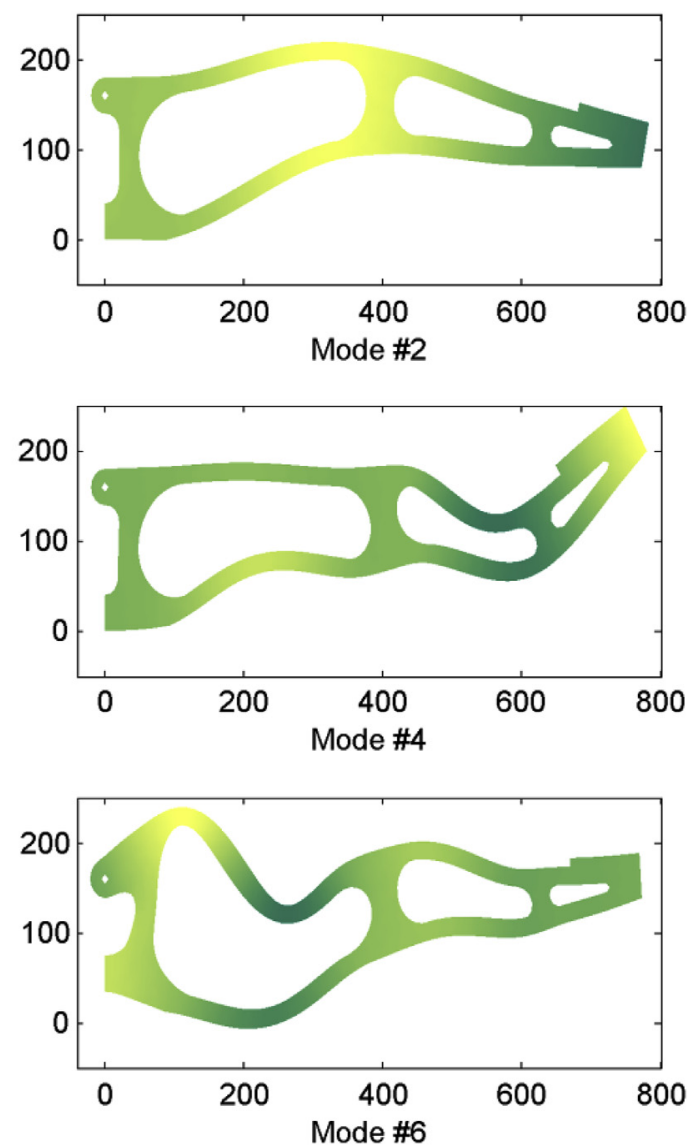

Fig. 13. First six eigenmodes of the bracket obtained by using the CQ4 element. Displacements have been multiplied by a factor of 40 while plotting the mode shapes. 


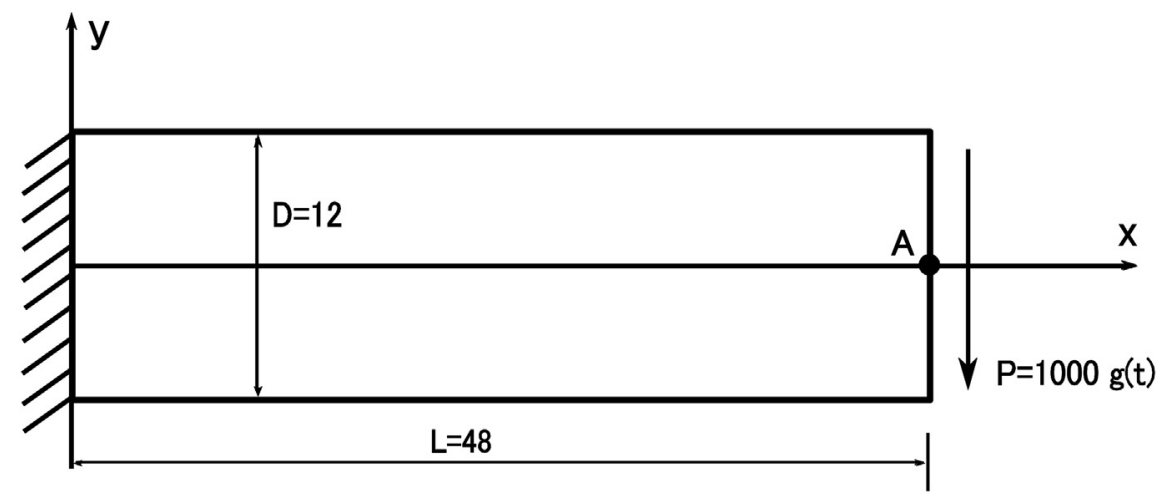

Fig. 14. Schematic sketch of a cantilever beam subjected to a parabolic time-dependent traction at the right free edge.

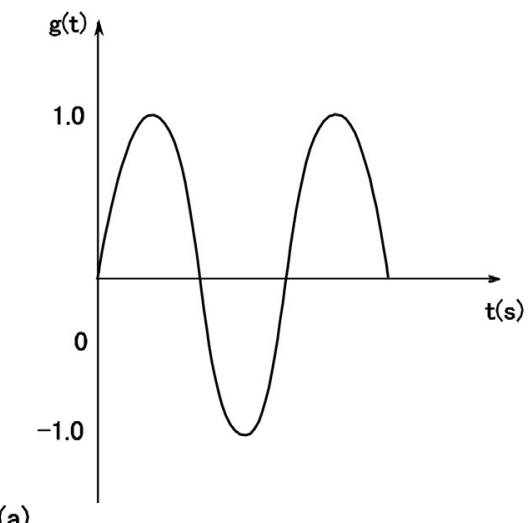

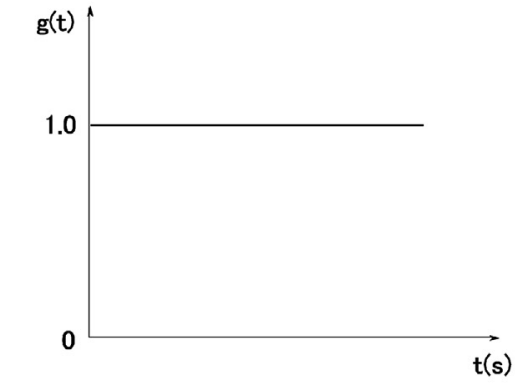

(b)

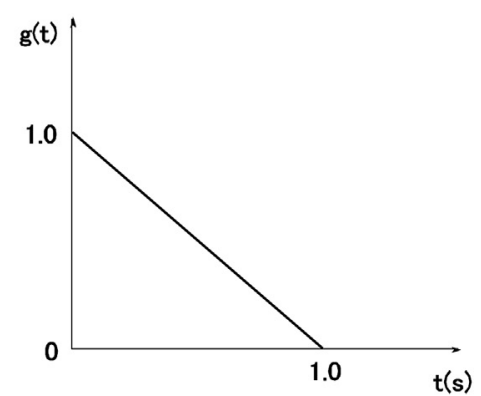

(c)

Fig. 15. Three functions $\mathrm{g}(\mathrm{t})$ considered for the forced vibration analysis; (a) harmonic load, (b) Heaviside step load, and (c) load affinely decreasing to zero in finite time.

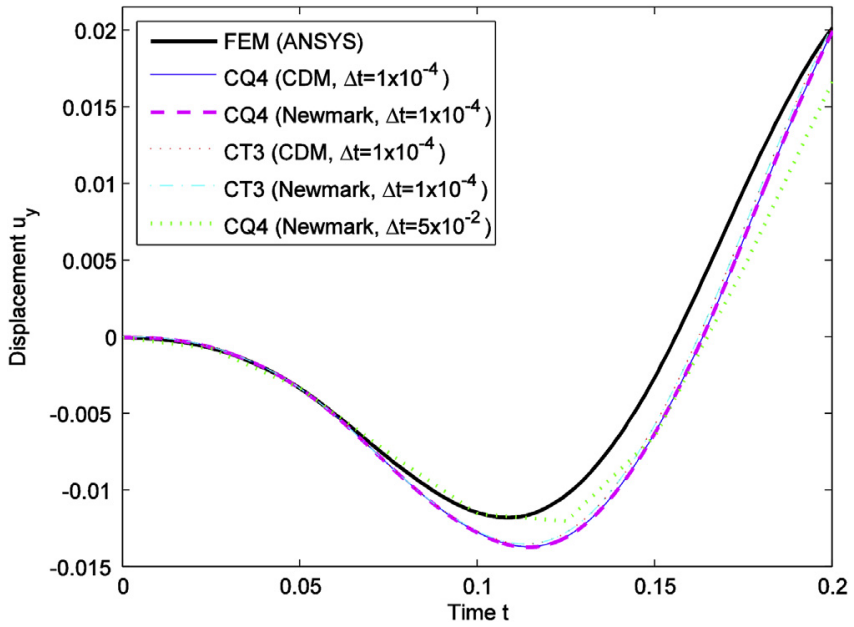

Fig. 16. Comparison of the time history of the displacement $u_{y}$ of point $\mathrm{A}$ obtained by using the CQ4 and the CT3 elements with that found by using the commercial FE software ANSYS under time-harmonic load. A structured fine FE mesh is employed for the analysis using ANSYS.

transverse isotropy. The kinematic relations for the strain $\varepsilon$ and the electric field $\mathbf{E}$ are

$\boldsymbol{\varepsilon}=\mathbf{L u} ; \mathbf{E}=-\operatorname{grad} \varphi$

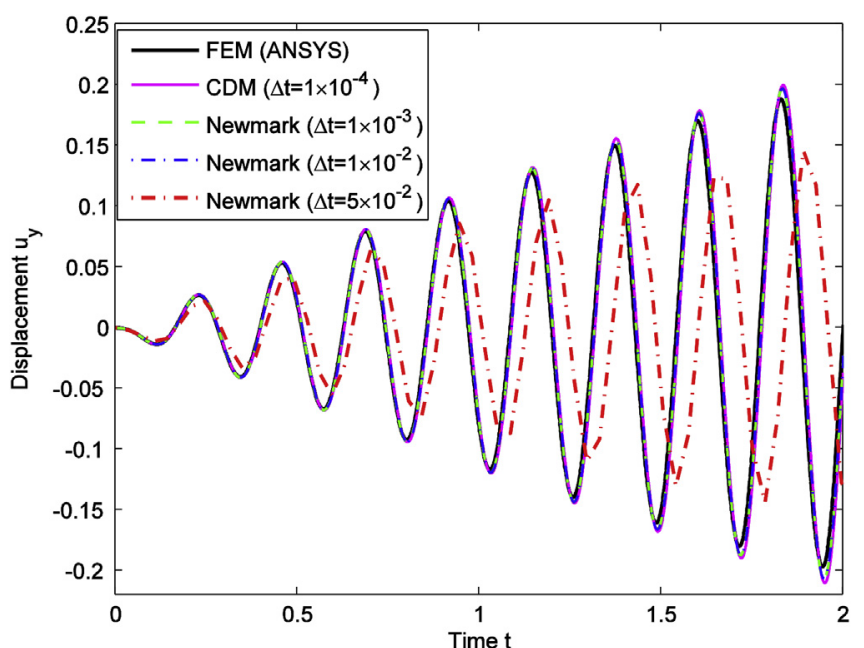

Fig. 17. For the time-harmonic load, time histories of the vertical displacement, $u_{y}$, of point A for different values of the time step size obtained by using the CQ4 element and the implicit and the explicit integration schemes.

Using basis functions for the CQ4 element, the displacement and the electric potential are approximated as

$\mathbf{u}(\mathbf{x})=\sum_{I=1}^{n_{s}} \tilde{\mathbf{N}}_{I}(\mathbf{x}) \mathbf{d}_{I} ; \varphi(\mathbf{x})=\sum_{I=1}^{n_{s}} \tilde{\mathbf{N}}_{I}(\mathbf{x}) \varphi_{I}$

where $\varphi$ is the electric potential. 


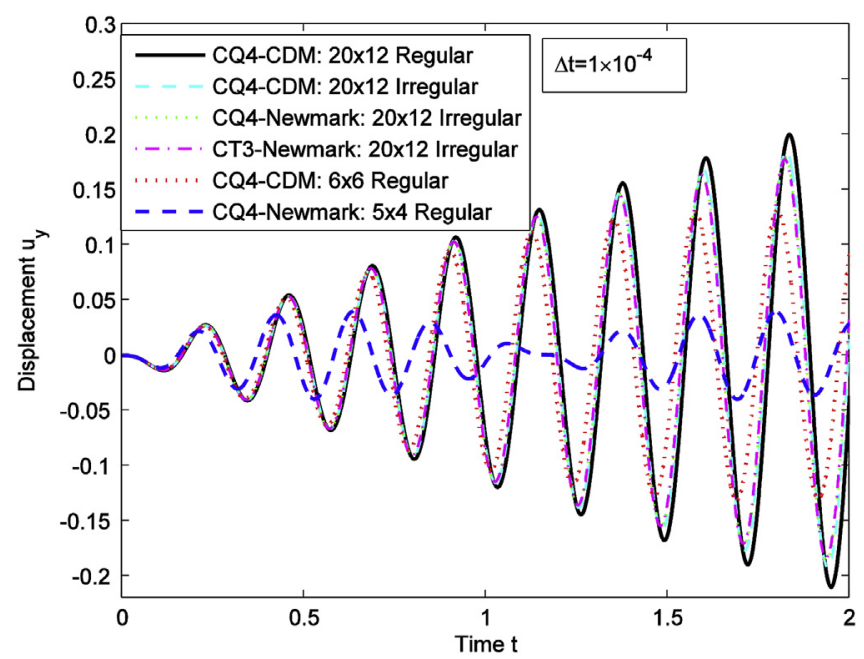

Fig. 18. Time histories of the vertical displacement of point A obtained by using the CQ4 and the CT3 elements for different structured and unstructured FE meshes under time-harmonic load. With the $20 \times 12 \mathrm{FE}$ mesh, differences in solutions with the structured and the unstructured meshes are insignificant for the CQ4 and the CT3 elements using either the Newmark or the CD methods. Very coarse FE meshes yield poor results.

Thus

$\boldsymbol{\varepsilon}=\mathbf{L u}=\sum_{I=1}^{n_{s}} \mathbf{B}_{u I} \mathbf{d}_{I} ; \mathbf{E}=-\operatorname{grad} \varphi=-\sum_{I=1}^{n_{s}} \mathbf{B}_{\varphi I} \varphi_{I}$

where

$\mathbf{B}_{u I}=\left[\begin{array}{ccccc}\tilde{N}_{I, x} & 0 & \cdots & \tilde{N}_{n_{s}, x} & 0 \\ 0 & \tilde{N}_{I, y} & \cdots & 0 & \tilde{N}_{n_{s}, y} \\ \tilde{N}_{I, y} & \tilde{N}_{I, x} & \cdots & \tilde{N}_{n_{s}, y} & \tilde{N}_{n_{s}, x}\end{array}\right] ; \mathbf{B}_{\varphi I}=\left[\begin{array}{ccc}\tilde{N}_{I, x} & \cdots & \tilde{N}_{n_{s}, x} \\ \tilde{N}_{I, y} & \cdots & \tilde{N}_{n_{s}, y}\end{array}\right]$

It is important to point out here that Eq. (34) is written in rectangular Cartesian coordinates. However, for studying axisymmetric deformations in cylindrical coordinates, Eq. (34) becomes

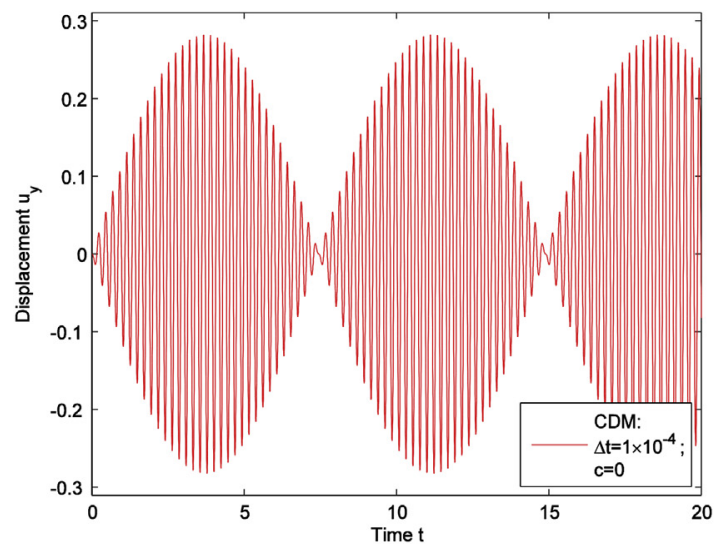

(a)

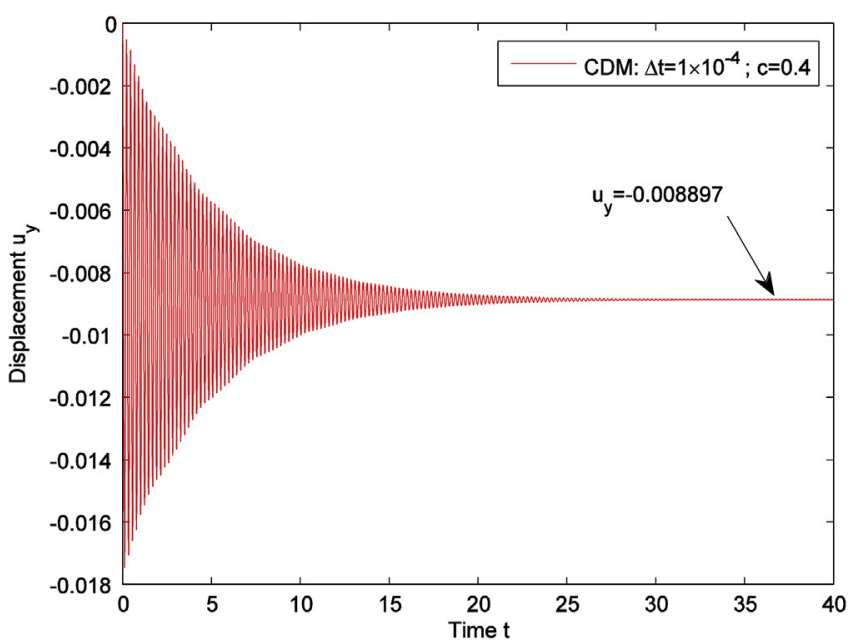

Fig. 20. Time history of the vertical deflection of point A of the beam using the explicit central difference method and damping coefficient, $c=0.4$.

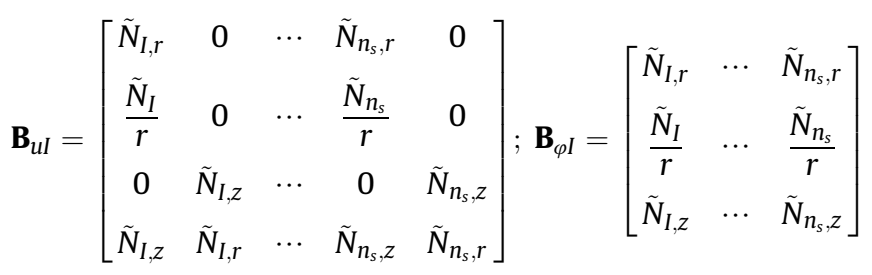

Following the standard approach, the discrete equations for piezoelectric materials without considering damping and external loads and charges can be expressed as

$\mathbf{M}_{u u} \ddot{\mathbf{d}}+\mathbf{K}_{u u} \mathbf{d}+\mathbf{K}_{u \varphi} \varphi=0$

$\mathbf{K}_{\phi u} \mathbf{d}-\mathbf{K}_{\phi \phi} \phi=0$

where the mass matrix $\mathbf{M}_{u u}$ is given by Eq. (13) and

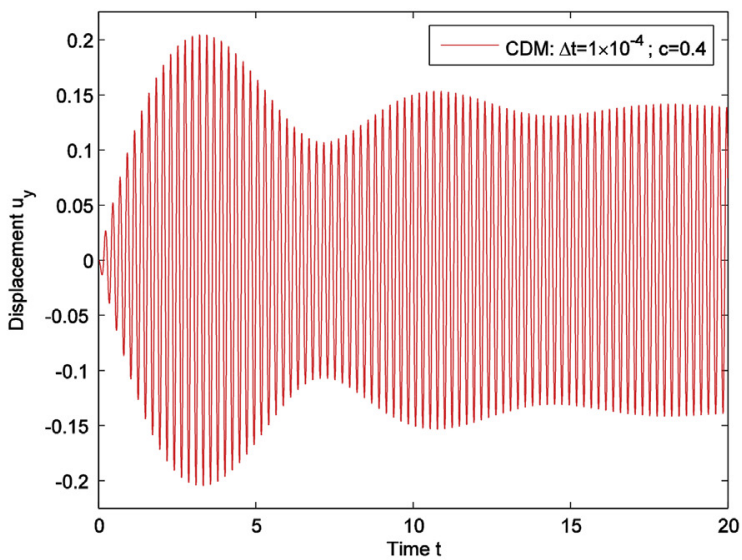

(b)

Fig. 19. Time history of the deflection of point A using the CDM for the time-harmonic load without (a) and with (b) damping $\mathrm{c}=0.4$. 


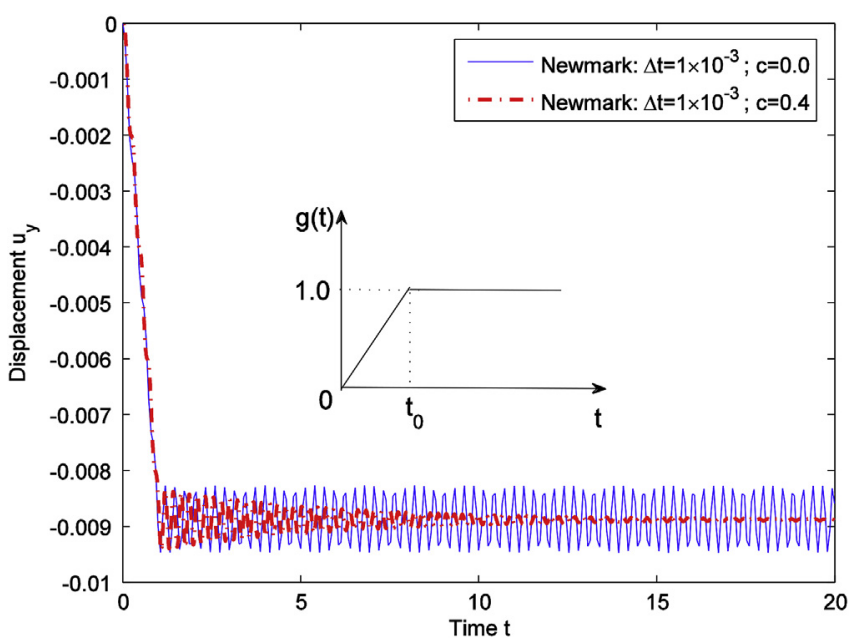

Fig. 21. Time histories of the vertical deflection of point $A$ of a beam using the implicit integration scheme with $\mathrm{c}=0.4$, and $\mathrm{c}=0.0$.

$$
\begin{aligned}
\mathbf{K}_{u u} & =\int_{\Omega} \mathbf{B}_{u}^{T} \mathbf{c}_{E} \mathbf{B}_{u} d \Omega ; \mathbf{K}_{u \varphi}=\int_{\Omega} \mathbf{B}_{u}^{T} \mathbf{e}^{T} \mathbf{B}_{\varphi} d \Omega ; \\
\mathbf{K}_{\varphi \varphi} & =-\int_{\Omega} \mathbf{B}_{\varphi}^{T} \kappa_{S} \mathbf{B}_{\varphi} d \Omega
\end{aligned}
$$

Solving Eq. (37) for the electric potential degrees of freedom, $\varphi=\mathbf{K}_{\varphi \varphi}^{-1} \mathbf{K}_{\varphi u} \mathbf{d}$, and then substituting the result into Eq. (36), we get the following structural equation

$\mathbf{M}_{u u} \ddot{\mathbf{d}}+\tilde{\mathbf{K}} \mathbf{d}=\mathbf{0}$

where

$$
\tilde{\mathbf{K}}=\mathbf{K}_{u u}+\mathbf{K}_{u \varphi} \mathbf{K}_{\varphi \varphi}^{-1} \mathbf{K}_{\varphi u}
$$

is the condensed electroelastic stiffness matrix. Eq. (39) is solved for the natural frequency in the same say as before.

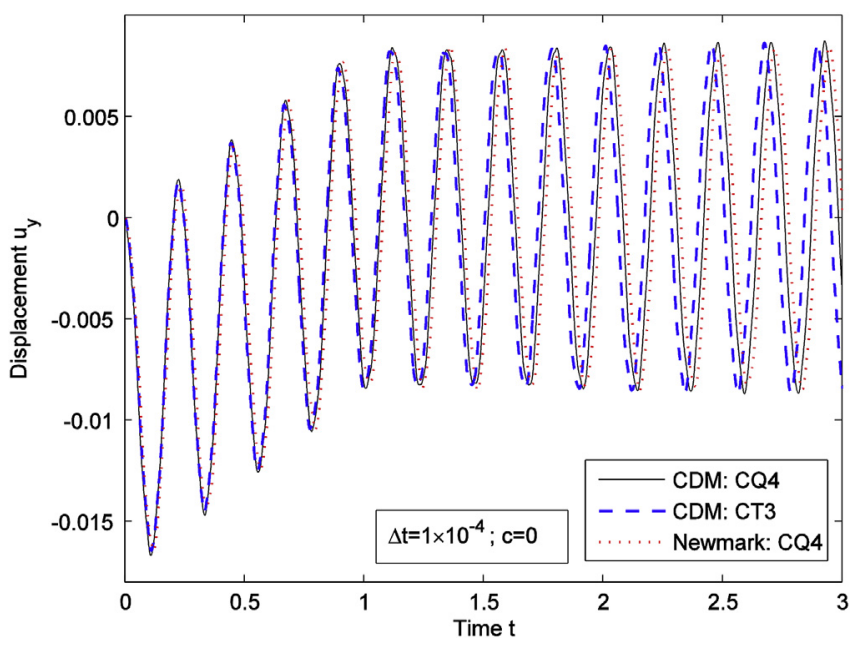

Fig. 22. Time histories of the displacement $u_{y}$ of point $\mathrm{A}$ without damping under transient load of $1 \mathrm{~s}$ duration.

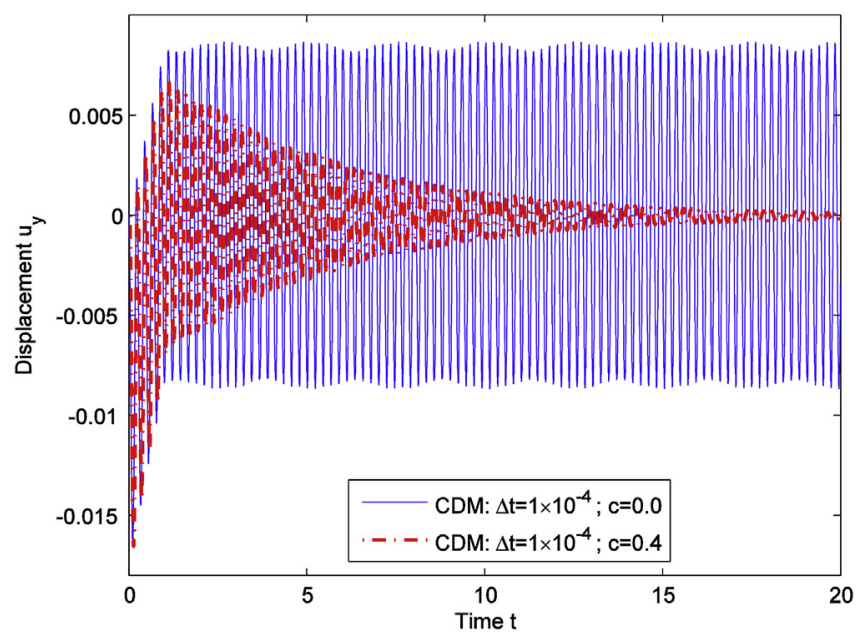

Fig. 23. Time histories of the displacement $u_{y}$ of point A using the explicit central difference method with $(c=0.4)$ and without $(c=0.0)$ damping under transient load of $1 \mathrm{~s}$ duration. These time histories agree well with those reported in $[7,13,17]$.

\subsection{Free vibrations of a piezoelectric disc}

We study free vibrations of a piezoelectric disc, depicted in Fig. 26, of diameter $3.2 \mathrm{~mm}$ and thickness $0.4 \mathrm{~mm}$ that has also been studied in (Bui et al., 2011a,b; Liu et al., 2013a,b). Values of material parameters for the PZT-4 disc are listed in Table 10. We consider two scenarios one when both surfaces are fully electroded and the other with only one surface electroded. Thus both resonant and anti-resonant frequencies can be computed. We compare our results with the literature values obtained by using the FEM (Liu et al., 2013a,b), the RPIM (Liu et al., 2013a,b) and the MK (Bui et al., 2011a,b). The presently computed first four natural frequencies with the CQ4 element for a coarse $(17 \times 3$ elements $)$ and a fine ( $32 \times 5$ elements) FE mesh are listed in Table 11. It is evident that the use of the CQ4 element gives very good values of the first

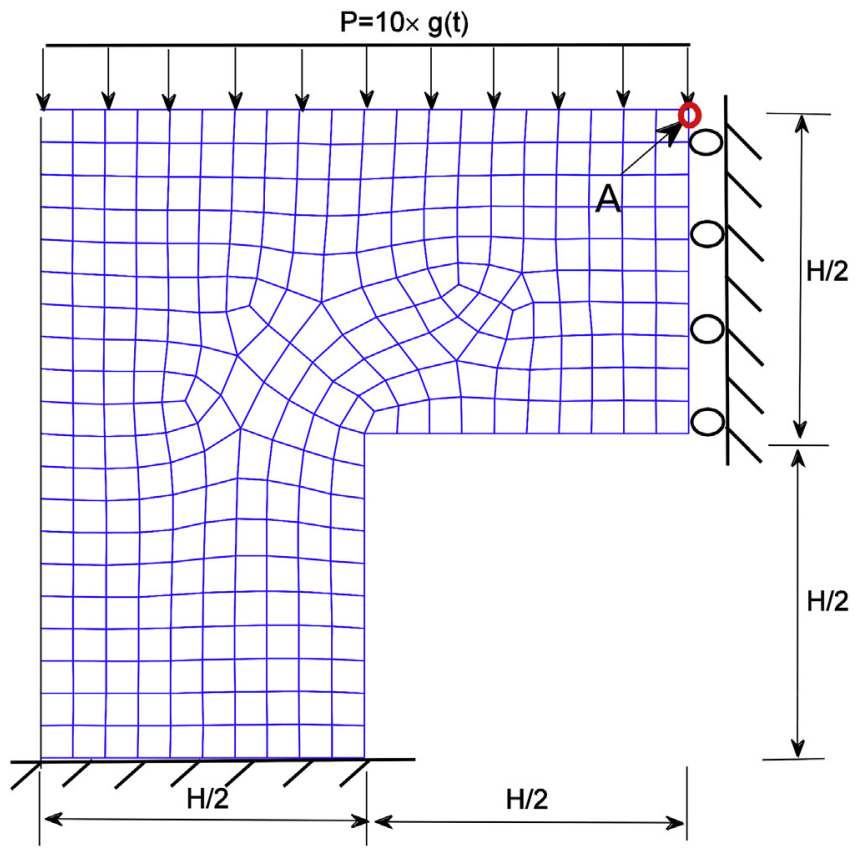

Fig. 24. Geometry of the L-shape panel, boundary conditions and its discretization into an unstructured FE mesh. 


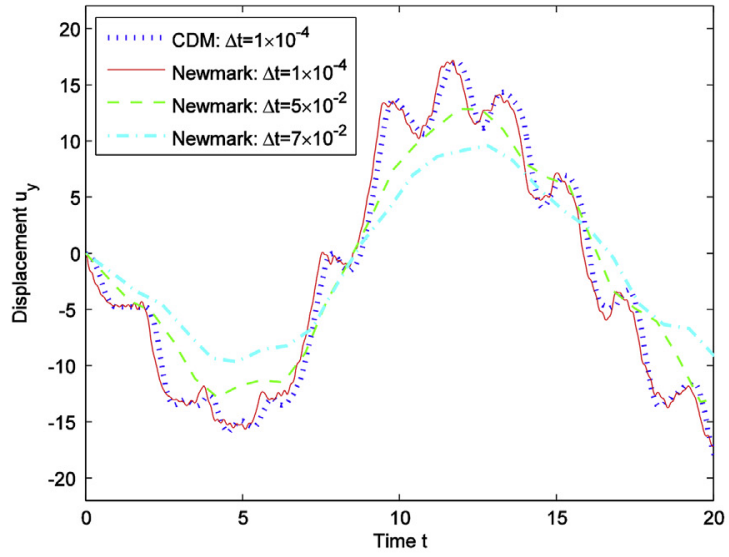

(a)

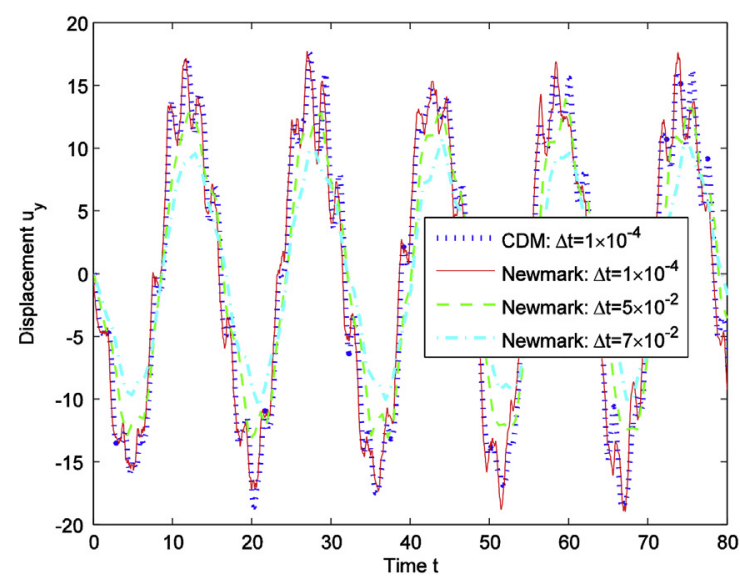

(b)

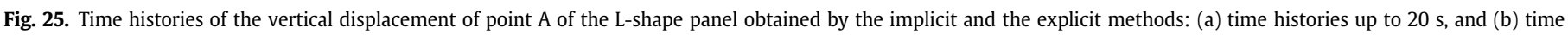
histories up to $80 \mathrm{~s}$.

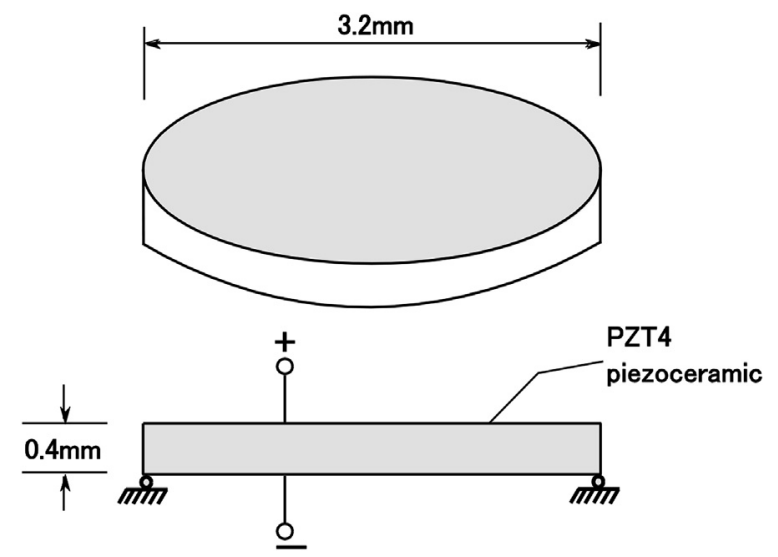

Fig. 26. Geometry of a typical piezoelectric disc. four natural frequencies for both cases when either both surfaces or only one surface is electroded. The corresponding mode shapes are shown in Fig. 27.

\subsection{A piezoelectric transducer}

The last example problem studied is that of the eigenvalue analysis of a piezoelectric transducer exhibited in Fig. 28 (Bui et al., 2011a,b; Liu et al., 2013a,b). The transducer made of a PZT4 ceramic wall with brass end caps and electrodes on the inner and the outer surfaces is assumed to undergo axisymmetric deformations. Values of material parameters used for the brass are: $E=104 \mathrm{GPa}, \nu=0.37$ and $\rho=8500 \mathrm{~kg} / \mathrm{m}^{3}$. Vibrations under open-circuited voltages with the electric potential on the inner surface equal to zero are analyzed; thus the anti-resonance (Liu et al., 2013a,b) frequencies are found. The first four natural frequencies obtained with the CQ4

Table 10

Material constants of PZT-4 ( $\left.\rho=7500 \mathrm{~kg} / \mathrm{m}^{3}\right)$ (Bui et al., 2011a; Liu et al., 2013a).

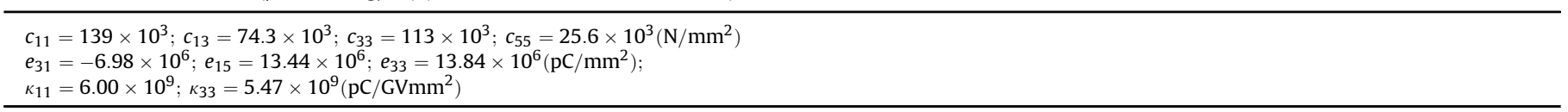

Table 11

Comparison of resonant and anti-resonant frequencies $(\mathrm{kHz})$ computed with different methods for coarse and fine meshes.

\begin{tabular}{|c|c|c|c|c|c|c|}
\hline Approach & Node density & Mode type & Mode 1 & Mode 2 & Mode 3 & Mode 4 \\
\hline \multirow[t]{4}{*}{ CAX4E (Liu et al., 2013a,b) } & \multirow[t]{2}{*}{$17 \times 3$} & Resonant & 41.9 & 166.3 & 344.7 & 489.9 \\
\hline & & Anti-resonant & 43.1 & 169.9 & 347.7 & 514.8 \\
\hline & \multirow[t]{2}{*}{$33 \times 5$} & Resonant & 43.0 & 172.7 & 354.1 & 481.3 \\
\hline & & Anti-resonant & 43.4 & 174.6 & 360.1 & 484.9 \\
\hline \multirow[t]{4}{*}{ RPIM (Liu et al., 2013a,b) } & \multirow{2}{*}{$17 \times 3$} & Resonant & 45.9 & 182.1 & 377.9 & 525.6 \\
\hline & & Anti-resonant & 46.4 & 184.2 & 383.8 & 530.9 \\
\hline & \multirow[t]{2}{*}{$33 \times 5$} & Resonant & 44.6 & 177.1 & 363.2 & 494.9 \\
\hline & & Anti-resonant & 45.0 & 179.1 & 368.6 & 499.1 \\
\hline \multirow[t]{4}{*}{ MK (Bui et al., 2011a,b) } & \multirow[t]{2}{*}{$17 \times 3$} & Resonant & 44.25 & 180.72 & 375.03 & 491.17 \\
\hline & & Anti-resonant & 46.83 & 184.13 & 378.76 & 553.51 \\
\hline & \multirow[t]{2}{*}{$33 \times 5$} & Resonant & 43.06 & 176.83 & 364.12 & 469.78 \\
\hline & & Anti-resonant & 45.55 & 180.12 & 366.87 & 519.06 \\
\hline \multirow[t]{4}{*}{ CQ4 } & \multirow[t]{2}{*}{$17 \times 3$} & Resonant & 44.87 & 180.22 & 376.19 & 505.74 \\
\hline & & Anti-resonant & 45.65 & 183.71 & 380.01 & 515.01 \\
\hline & \multirow[t]{2}{*}{$33 \times 5$} & Resonant & 43.13 & 176.78 & 364.92 & 487.33 \\
\hline & & Anti-resonant & 44.52 & 179.65 & 368.36 & 493.11 \\
\hline
\end{tabular}



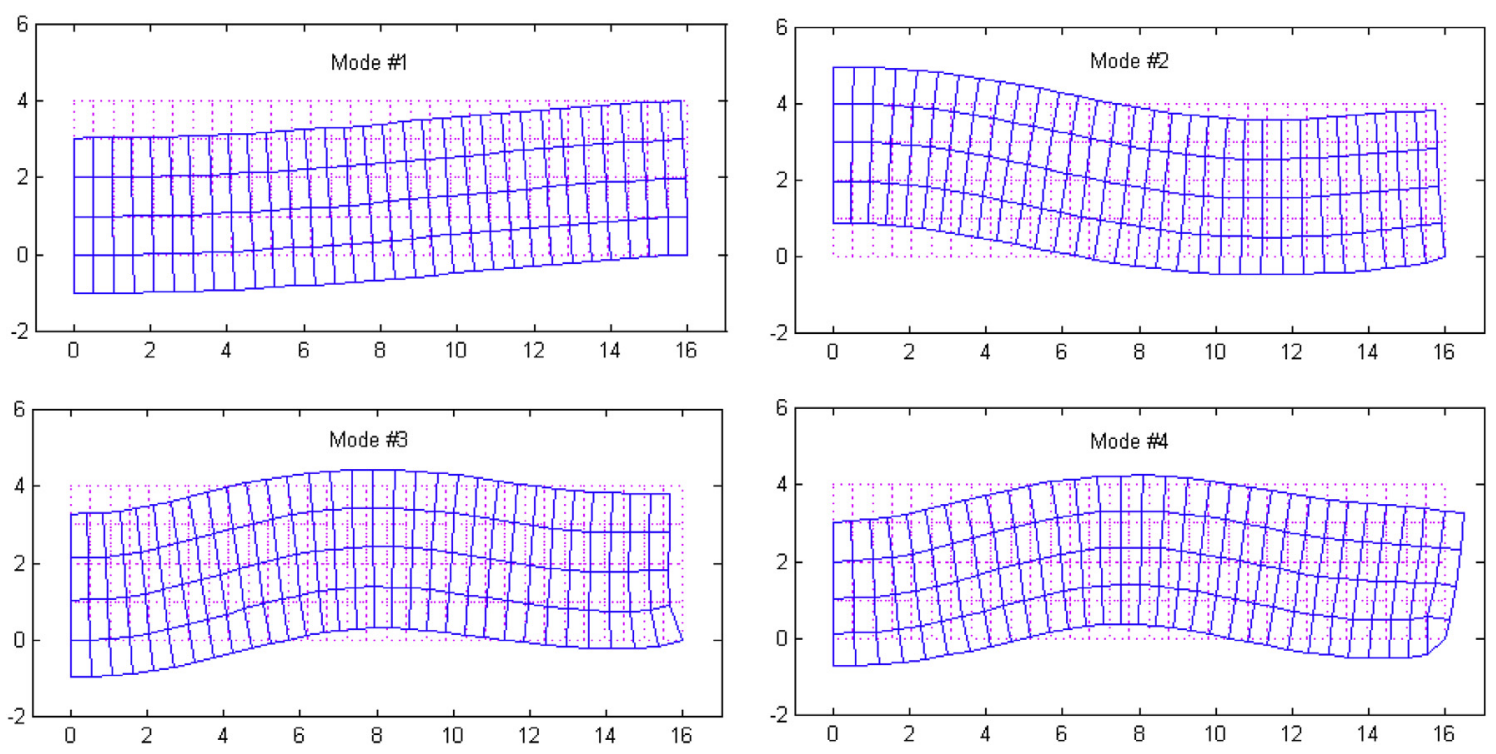

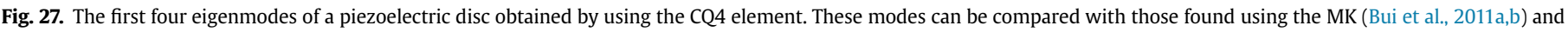
the RPIM (Liu et al., 2013a,b) methods. Displacements have been multiplied by a factor of 40 .

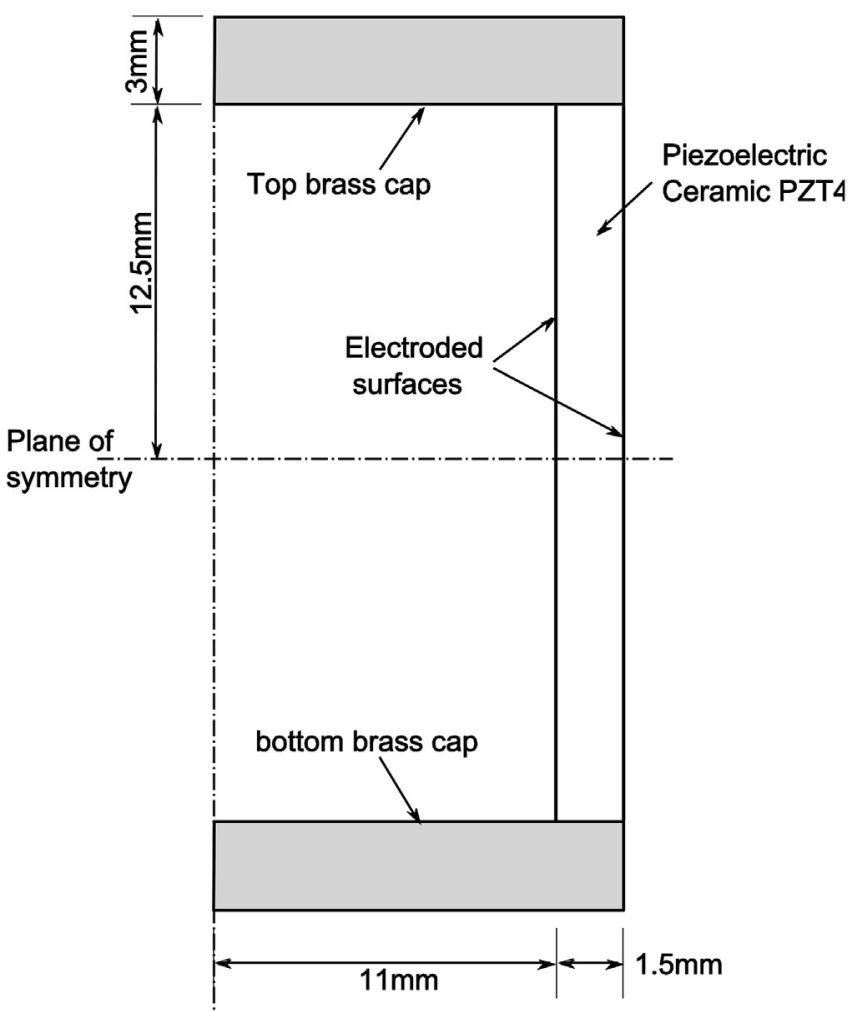

Axis of symmetry

Fig. 28. Geometry of a piezoelectric transducer. (for 136 and 176 elements) and the conventional Q4 (176 elements) elements and their percentage differences from the corresponding values are listed in Table 12 . The maximum difference of $17 \%$ between the experimental (Liu et al., 2013a,b) and the frequencies computed with 176 elements confirms that the CQ4 element performs well in eigenvalue analysis of piezoelectric structures, and gives more accurate frequencies than those obtained with the standard Q4 element. The first four eigenmodes are displayed in Fig. 29.

\section{Conclusions}

We have used the basis functions for the CQ4 element and the finite element method to analyze free and forced twodimensional (either plane stress or axisymmetric) vibrations of several linear elastic structures of complex shapes, and free vibrations of two linear piezoelectric structures. In each case the frequencies, the mode shapes and the displacements are found to agree well either with the corresponding experimental results or with the literature results obtained by other methods. Generally, the basis functions for the CT3 and the CQ4 elements provide better numerical solution than that computed with the traditional T3 and Q4 elements. For two of the problems studied, results computed with the CQ4 and the traditional Q8 elements are very close to each other. The computed response for the forced vibration problems using either the implicit or the explicit method is found to be quite accurate. The CT3 and the CQ4 elements can be easily implemented in existing finite element software.

Table 12

The first four eigenvalues $(\mathrm{kHz})$ and percentage errors for a piezoelectric transducer obtained by using 136 and 176 CQ4 elements.

\begin{tabular}{|c|c|c|c|c|c|}
\hline \multicolumn{2}{|c|}{ Approach } & Mode 1 & Mode 2 & Mode 3 & Mode 4 \\
\hline \multicolumn{2}{|c|}{ Experiment (Liu et al., 2013a,b) } & 18.6 & 35.4 & 54.2 & 63.3 \\
\hline CQ4 & 136 elements & $\begin{array}{l}19.965 \\
(7.34 \%)\end{array}$ & $\begin{array}{l}42.043 \\
(18.76 \%)\end{array}$ & $\begin{array}{l}59.238 \\
(9.29 \%)\end{array}$ & $\begin{array}{l}67.432 \\
(6.53 \%)\end{array}$ \\
\hline & 176 elements & $\begin{array}{l}18.783 \\
(0.98 \%)\end{array}$ & $\begin{array}{l}41.227 \\
(16.46 \%)\end{array}$ & $\begin{array}{l}57.210 \\
(5.55 \%)\end{array}$ & $\begin{array}{l}65.109 \\
(2.86 \%)\end{array}$ \\
\hline Q4 & 176 elements & $\begin{array}{l}20.352 \\
(9.41 \%)\end{array}$ & $\begin{array}{l}42.872 \\
(21.11 \%)\end{array}$ & $\begin{array}{l}60.424 \\
(11.48 \%)\end{array}$ & $\begin{array}{l}67.988 \\
(7.41 \%)\end{array}$ \\
\hline
\end{tabular}



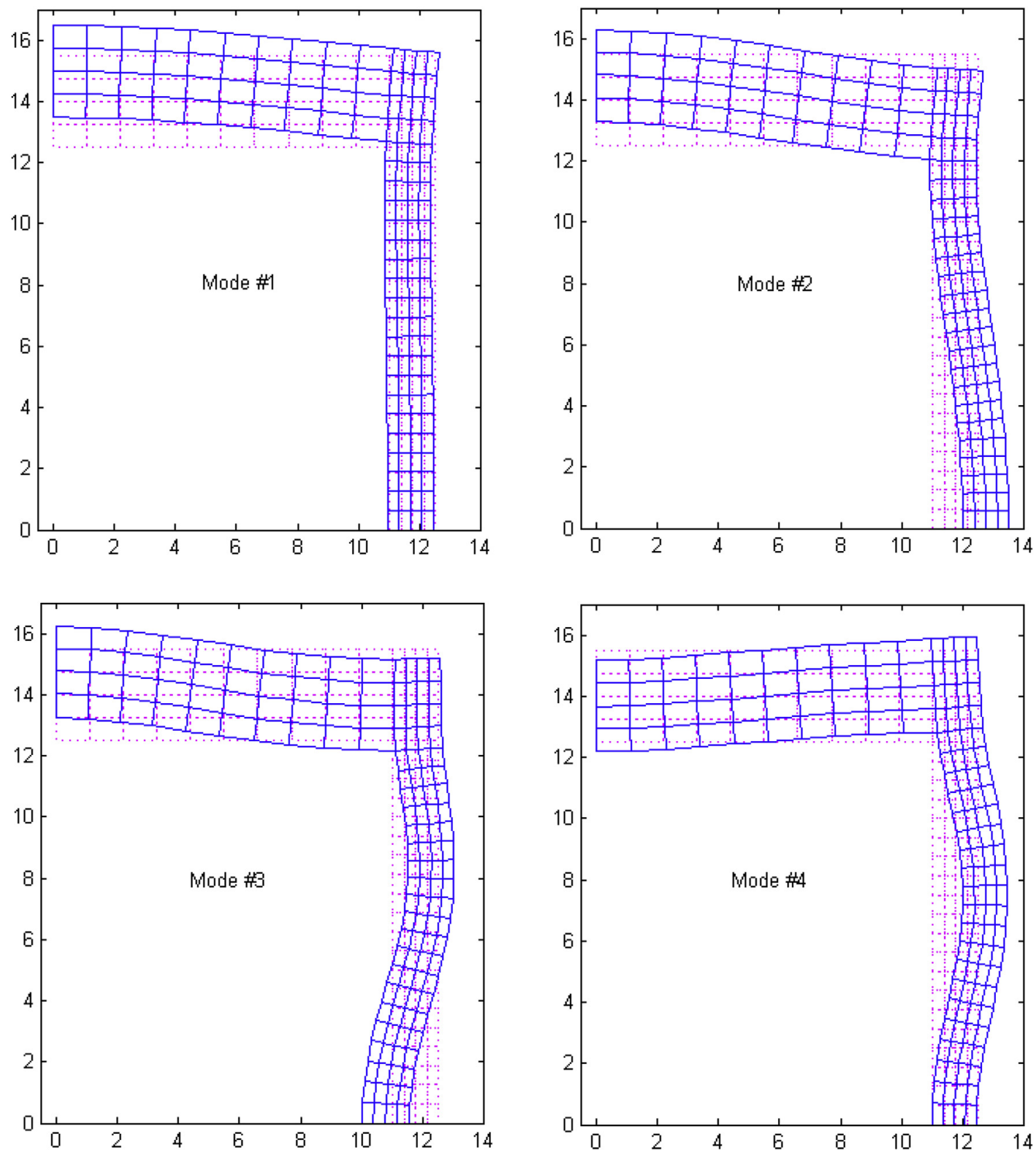

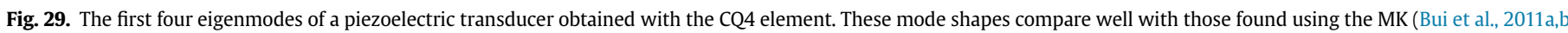
and the RPIM (Liu et al., 2013a,b) methods. Displacements have been multiplied by a factor of 40 .

\section{Acknowledgments}

The financial supports of the Grant-in-Aid for Scientific Research (No. 26-04055) - Japan Society for the Promotion of Sciences (JSPS, ID No. P14055) and the National Natural Science Foundation of China (Grant No. 51208453) are gratefully acknowledged. RCB's work was partially supported by the US Office of Naval Research grant N00014-11-1-0594 to Virginia Polytechnic Institute and State University with Dr. Y. D. S. Rajapakse as the Program Manager.

\section{References}

Allik, H., Hughes, R.J.T., 1970. Finite element method for piezoelectric vibration. Int. J. Numer. Methods Eng. 2, 151-157.

Batra, R.C., Liang, X.Q., Yang, J.S., 1996. Shape Control of Vibrating Simply Supported Plates. AIAA J. 34, 116-122.
Batra, C.R., Geng, T.S., 2001. Enhancement of the dynamic buckling load for a plate by using piezoceramic actuators. Smart Mater. Struct. 10, 925-933.

Batra, C.R., Liang, Q.X., 1997. Finite dynamic deformations of smart structures. Comp. Mech. 20, 427-438.

Batra, C.R. Geng T.S, 2002. Comparison of active constrained layer damping by using extension and shear mode actuators. J. Intell. Mater. Struct. 13, 349-367.

Benjeddou, A., 2000. Advances in piezoelectric finite element modeling of adaptive structural elements: a survey. Comput. Struct. 76, 347-363.

Brebbia, C.A., Telles, J.C., Wrobel, L.C., 1984. Boundary Element Techniques. Berlin Springer Verlag.

Bui, Q.T., Khosravifard, A., Zhang, Ch, Hematiyan, R.M., Golub, M.V., 2013. Dynamic analysis of sandwich beams with functionally graded core using a truly meshfree radial point interpolation method. Eng. Struct. 47, 90-104.

Bui, Q.T., Nguyen, N.M., Zhang, Ch, Pham, D.A.K., 2011a. An efficient meshfree method for analysis of two-dimensional piezoelectric structures. Smart Mater. Struct. 20, 065016.

Bui, Q.T., Nguyen, N.M., Zhang, Ch, 2011b. A moving Kriging interpolation-based element-free Galerkin method for structural dynamic analysis. Comput. Methods Appl. Mech. Eng. 200, 1354-1366. 
Bui, Q.T., Nguyen, N.M., 2011. A moving Kriging interpolation-based meshfree method for free vibration analysis of Kirchoff plates. Comput. Struc. 89, 380-394.

Bui, Q.T., Vo, Q.D., Zhang, Ch, Nguyen, D.D., 2014. A consecutive-interpolation quadrilateral element (CQ4): formulation and applications. Finite Elem. Anal. Des. 84, 14-31.

Bui, Q.T., Zhang, Ch, 2013. Analysis of generalized dynamic intensity factors of cracked magnetoelectroelastic solids by X-FEM. Finite Elem. Anal. Des. 69, 19-36.

Bui, Q.T., Zhang, Ch, 2012. Extended finite element simulation of stationary dynamic cracks in piezoelectric solids under impact loading. Comput. Mater. Sci. 62, $243-257$.

Cottrell, J.A., Reali, A., Bazilevs, Y., Hughes, J.R.T., 2006. Isogeometric analysis of structural vibrations. Comput. Methods Appl. Mech. Eng. 195, 5257-5296.

Cui, X.Y., Liu, G.R., Li, Y.G., 2010. A cell-based smoothed radial point interpolation method (CS-RPIM) for static and free vibration of solids. Eng. Anal. Bound. Elem. 34, 144-157.

Dai, K.Y., Liu, G.R., 2007. Free and forced vibration analysis using the smoothed finite element method (SFEM). J. Sound. Vib. 301, 803-820.

Gu, Y.T., Liu, G.R., 2005. A meshfree weak-strong (MWS) form method for time dependent problems. Comput. Mech. 35, 134-145.

Gu, Y.T., Liu, G.R., 2001. A meshless local Petrov-Galerkin (MLPG) method for free and forced vibration analyses for solids. Comput. Mech. 27, 188-198.

Kang, Z., Bui, Q.T., Nguyen, D.D., Saitoh, Hirose S., 2015. An extended consecutiveinterpolation quadrilateral element (XCQ4) applied to linear elastic fracture mechanics. Acta Mech. 226, 3991-4015.

Kosta, T., Tsukanov, I., 2014. Meshfree natural vibration analysis of 2D structures. Comput. Mech. 53, 283-296.

Li, H., Wang, Q.X., Lam, K.Y., 2004. Development of a novel meshless Local Kriging (LoKriging) method for structural dynamic analysis. Comput. Methods Appl. Mech. Eng. 193, 2599-2619.

Liu, G.R., Dai, K.Y., Lim, M.K., Gu, Y.T., 2013a. A radial point interpolation method for simulation of two-dimensional piezoelectric structures. Smart Mater. Struct. 12 $171-180$.
Liu, P., Yu, T.T., Bui, Q.T., Zhang, Ch, Xu, Y., Lim, C.W., 2014. Transient thermal shock fracture analysis of functionally graded piezoelectric materials by the extended finite element method. Int. J. Solids Struct. 51, 2167-2182.

Liu, P., Yu, T.T., Bui, Q.T., Zhang, Ch, 2013b. Transient dynamic crack analysis in nonhomogeneous functionally graded piezoelectric materials by the X-FEM. Comput. Mater. Sci. 69, 542-558.

Manolis, G.D., Beskos, E.D., 1988. Boundary Element Methods in Elastodynamics. Unwin Hyman, London.

Miranda, D.S., Molari, L. Ubertini, F., 2008. A consistent approach for mixed stress finite element formulations in linear elastodynamics. Comput. Methods Appl. Mech. Eng. 197, 1376-1388.

Petyt, M., 2010. Introduction to Finite Element Vibration Analysis, second ed Cambridge University Press, Cambridge, UK.

Sadeghirad, A., Kani, M.I., Rahimian, M., Astaneh, V.A., 2009. A numerical approach based on the meshless collocation method in elastodynamics. Acta Mech. Sin. 25, 857-870.

Sharma, K., Bui, Q.T., Zhang, Ch, Bhargava, R.R., 2013. Analysis of a subinterface crack in piezoelectric bimaterials with the extended finite element method. Eng. Fract. Mech. 104, 114-139.

Timoshenko, S.P. Goodier, J.N., 1970. Theory of Elasticity. McGraw-Hill, New York.

Valizadeh, N., Bui, Q.T., Vu, T.V., Thai, H.T., Nguyen, N.M., 2013. Isogeometric simulation for buckling, free and forced vibration of orthotropic plates. Int. J Appl. Mech. 05, 1350017.

Yang, S.J., Batra, C.R., 1994. Free vibrations of a piezoelectric body. J. Elast. 34 239-254.

Zhao, C., Steven, G.P., 1996. Asymptotic solutions for predicted natural frequencies of two-dimensional elastic solid vibration problems in finite element analysis. Int. J. Numer. Methods Eng. 39, 2821-2835.

Zheng, C., Wu, S.C., Tang, X.H., Zhang, J.H., 2010. A novel twice-interpolation finite element method for solid mechanics problems. Acta Mech. Sin. 26, 265-278.

Zienkiewicz, O.C., Taylor, R.L., Zhu, J.Z., 2005. The Finite Element Method: its Basis and Fundamentals, sixth ed. Elsevier Butterworth-Heinemann, MA. 